BNL 52298

\title{
FUNDAMENTALS OF PARTICLE BEAM DYNAMICS AND PHASE SPACE
}

\author{
W.T. Weng and S.R. Mane

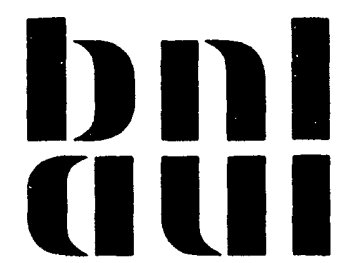

September 4, 1991

ALTERNATING GRADIENT SYNCHROTRON DEPARTMENT

BROOKHAVEN NATIDNAL LABORATORY, ASSOCIATED UNIVERSITIES, INC.

UPTON, NEW YORK 11973

UNDER CONTRACT NO. DE-AC02-76CH00016 WITH THE UNITED STATES DEPARTMENT OF ENERGY 


\title{
FUNDAMENTALS OF PARTICLE BEAM DYNAMICS AND PHASE SPACE
}

\author{
W.T. Weng and S.R. Mane
}

September 4, 1991

ALTERNATING GRADIENT SYNCHROTRON DEPARTMENT BROOKHAVEN NATIONAL LABORATORY ASSOCIATED UNIVERSITIES, INC. UPTON, LONG ISLAND, NEW YORK 11973 


\section{DISCLAIMER}

This report was prepared as an account of work sponsored by an agency of the United States Government. Neither the United States Government nor any agency thereof. nor any of their employees. nor any of their contractors, subcontractors. or their employees, makes any warranty, express or implied, or assumes any legal liability or responsibility for the accuracy. completeness, or usefulness of any information. apparatus, product, or process disclosed. or represents that its use would not infringe privately owned rights. Reference herein to any specitic ccmmercial product, process. or service by trade name, trademark, manufacturer, or otherwise, does not necessarily constitute or imply its endorsement, recommendat: in, or favoring by the United States Government or any agency, contractor or stbcontractor thereof. The views and opinions of authors expressed herein do not necessarily state or reflect those of the United States Government or any agency, cont:actor or subcontractor thereof.

Printed in the United States of America Available from

National Technical Information Service

U.S. Department of Commerce

5285 Port Royal Road

Springfield, VA 22161

NTIS price codes:

Printed Copy: A03; Microfiche Copy: A01 


\title{
FUNDAMENTALS OF PARTICLE BEAM DYNAMICS AND PHASE SPACE*
}

\author{
W.T. Weng and S.R. Mane \\ Brookhaven National Laboratory, Upton, NY 11973
}

\section{Contents}

1 Introduction 2

2 Transverse Motion - Betatron Oscillations 5

2.1 Coordinate Description ................ 5

2.2 Hamiltonian Formulation . . . . . . . . . . . . . 12

3 Machine Lattice 14

3.1 Comments on Tune Choice . . . . . . . . . . . . . . . . 14

3.2 Orbit Distortion due to Field Error . . . . . . . . . . . . . 15

3.3 Amplitude Function Distortion due to Gradient Error . . . . . . . 16

3.4 Momentum Effects ... . . . . . . . . . . . . . 17

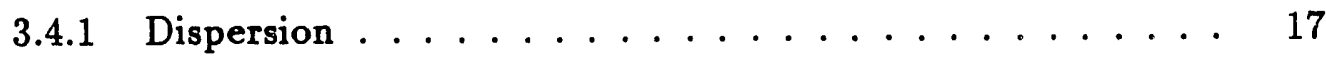

3.4 .2 Chromaticity ....................... 19

4 Representation of a Particle Beam 20

4.1 Introduction . . . . . . . . . . . . . . . 20

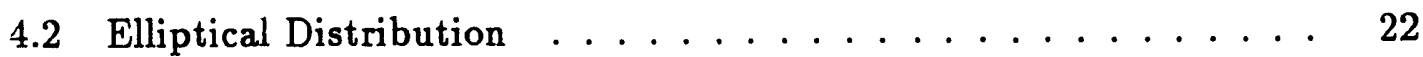

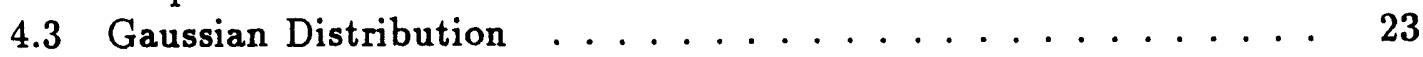

4.4 Emittance and Admittance . . . . . . . . . . . . . . 24

4.5 Emittance of a Beam .................. 25

4.6 Matching between Two Optical Channels . . . . . . . . . . 27

4.7 Liouville's Theorem . . . . . . . . . . . . . . . . . . . 28

4.8 Normalized Emittance . . . . . . . . . . . . . . 30

5 Longitudinal Motion - Synchrotron Oscillations 31

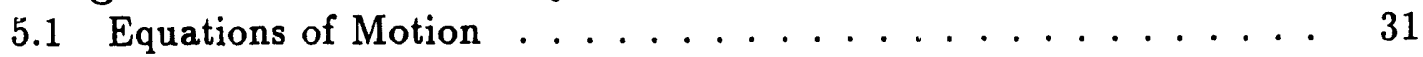

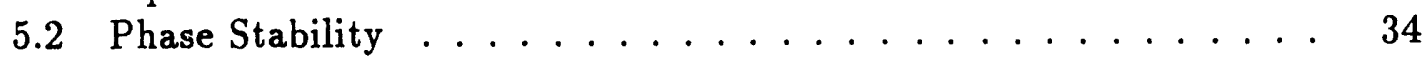

5.3 Transition Energy . . . . . . . . . . . . . . . 35

5.4 Hamiltonian Formulation . . . . . . . . . . . . . . 37

5.5 Adiabatic Damping .................. . . 41

5.6 Phase-space Matching during Synchronous Transfer . . . . . . . 41

6 Bibliography $4 \mathbf{4 3}$

-Work performed under the auspices of the U.S. Department of Energy. 
FUNDAMENTALS OF PARTICLE BEAM DYNAMICS AND PHASE SPACE

\author{
W.T. Weng and S.R. Mane \\ Brookhaven National Laboratory, Upton, NY 11973
}

\title{
1 Introduction
}

The first accelerator to achieve disintegration of atomic nuclei by accelerated particles was built by J.D. Cockroft and E.T. Walton in 1932. The accelerator produced proton beams with energy higher than $10 \mathrm{keV}$ for the first time. For that they were awarded the Nobel Prize in physics in 1951. Today accelerators are used in many areas of research and medical and industrial applications. In 1989, a new division, the Division of Particle Beams, was created within the APS to foster the development of beam physics and interactions among traditionally separated disciplines. For the early history of the development of particle accelerators, see Livingston [1]. For an overview of today's new accelerator projects, see Teng [2].

Accelerators can be broadly divided into linear or circular. In the former, the particles pass through the accelerator just once, whereas in the latter, they circulate around the circumference many times. Among all types of accelerators, we shall concentrate on the synchrotron for today's discussion (see Fig. 1.1). Briefly, a synchrotron is a circular accelerator with the following three characteristics:

1. Magnetic guide field (dipoles) and confinement components (quadrupoles) are placed in a small neighborhood of the equilibrium orbit.

2. Particles are kept in phase with the rf accelerating field through the acceleration cycle to achieve high energy.

3. Magnetic fields are varied adiabatically with the energy of the particles. 


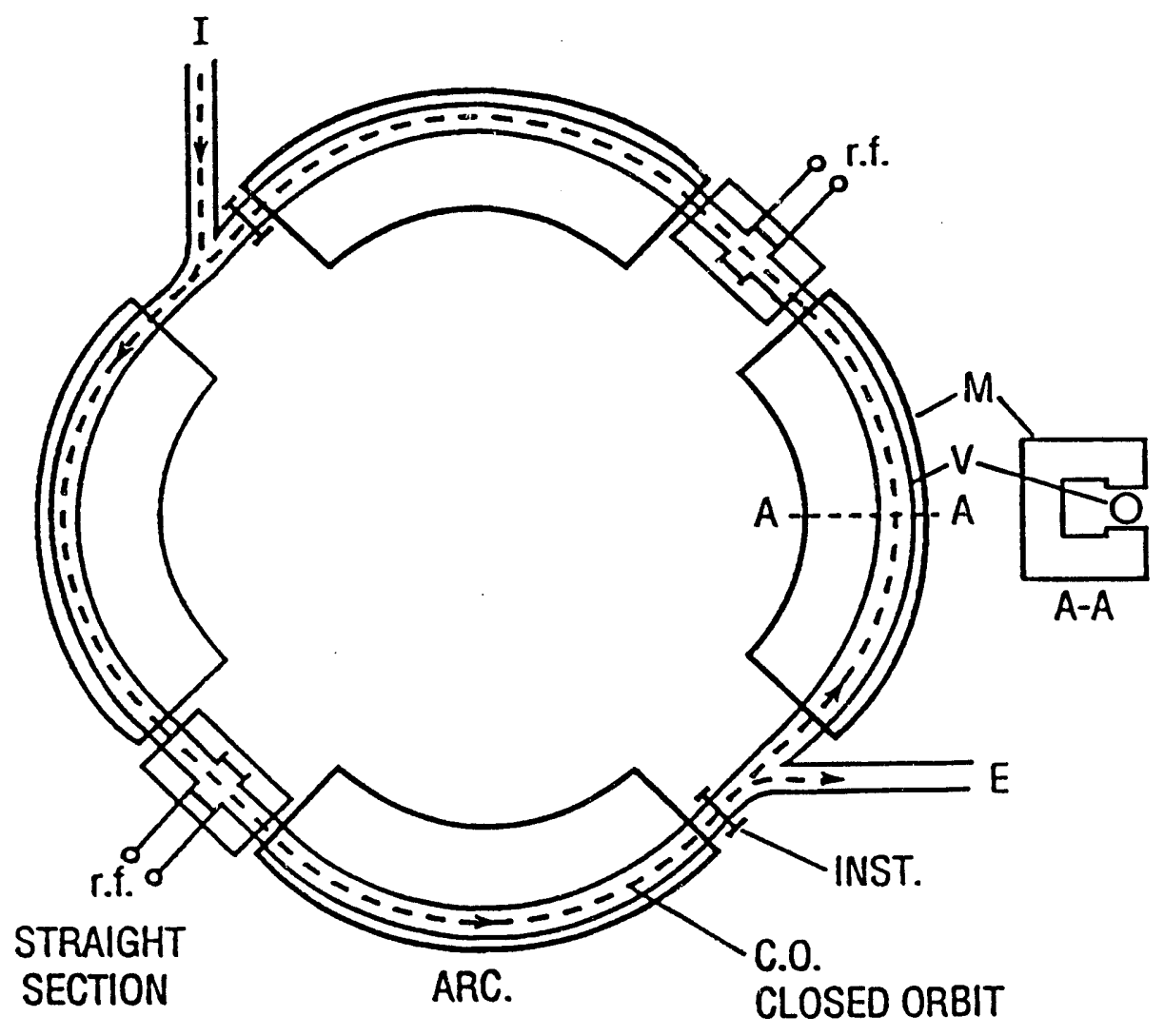

$M$ : MAGNET \& POWER SUPPLY

r.f.: RADIO - FREQUENCY ACCELERATION

$V$ : VACUUM CHAMBER

I : INJECTION

E: EXTRACTION

I \& $C:$ INSTRUMENTION AND CONTROL

Fig. 1.1 Schematics of a synchrotron.

Before we start formal discussion of the theory of synchrotrons, a few general remarks will help to define the relationship of this course to others given in this school.

a. A storage ring is an example of a synchrotron where the 
energy of the particles is held constant for a long time. Typically particles are injected into the accelerator at low energy, accelerated to high energy, and then held at the top energy for several hours for physics research. Thus our discussion below also applies to storage rings.

b. In a synchrotron, the ideal particle follows a path called the equilibrium orbit or closed orbit. The coordinates and momenta of an actual particle are usually expressed relative to the closed orbit. Then each particle has three degrees of freedom, which form a six-dimensional phase space. When the coupling among them can be neglected, it is conveniently separated into

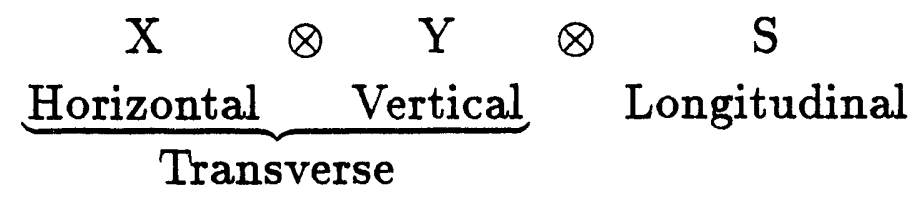

The longitudinal degree of freedom determines the length of a bunch, while the transverse degrees determine its height and width.

c. Coordinate vs. phase-space description: Rather than describing the trajectory of a particle as a function of time (by solving the equations of motion), it is sometimes preferable to describe a particle by specifying a point in phase space. We shall explain in more detail below. The phase-space description is useful when treating higher-order terms in the equations of motion. It is also useful when treating a distribution of particles. One can visualize a beam of particles as a "cloud" of points in phase space.

d. Single, multi-particle, interacting, particle description: In these lectures, we shall treat the individual particles as independent. Hence, in the equations of motion, we include only the influence of the electric and magnetic fields provided externally. We neglect interactions by the particles among themselves, e.g. their mutual repulsion, because they all have the same sign of charge.

e. Linear approximation and nonlinear terms: In general, even 
in the independent particle approximation, the equation of motion of a particle is nonlinear. We shall treat only linear equations of motion below.

\section{Transverse Motion - Betatron Oscillations}

\subsection{Coordinate Description}

We begin with the transverse degrees of freedom, which can be described by two coordinates $x$ and $y$. See also Ref. 3 for more details. Obviously, we take $x$ to be radially outward, and $y$ to be vertical. Because of the focusing, which is primarily due to the quadrupole magnetic fields, a typical particle will perform oscillations around the closed orbit, known as betatron oscillations (see Fig. 2.1). We can derive the equation of motion as follows. First, note that a particle obeys the Lorentz equation

$$
\frac{d \vec{P}}{d t}=\vec{F}=e\left(\vec{E}+\frac{\vec{v}}{c} \times \vec{B}\right),
$$

but this equation uses time as the independent variable. In accelerator physics it is more convenient to use the arc length $s$ along the reference trajectory, and to measure the particle coordinates and momenta as deviations from that of the ideal particle. Thus Eq. (2.1) must be rewritten in a new coordinate system. We assume that the reference trajectory lies in the horizontal plane. If $h(s)$ is the local curvature of the reference trajectory, then the total angle through which it bends, from $s_{0}$ to $s$, is

$$
\theta=\int_{s_{1}}^{s} h\left(s^{\prime}\right) d s^{\prime} .
$$

The solution for $\vec{P}$, as a function of $s$, can be formally written as

$$
\vec{P}(s)=\vec{P}\left(s_{0}\right)+\int_{s_{0}}^{s} F\left(s^{\prime}\right) \frac{d t}{d s^{\prime}} d s^{\prime}
$$




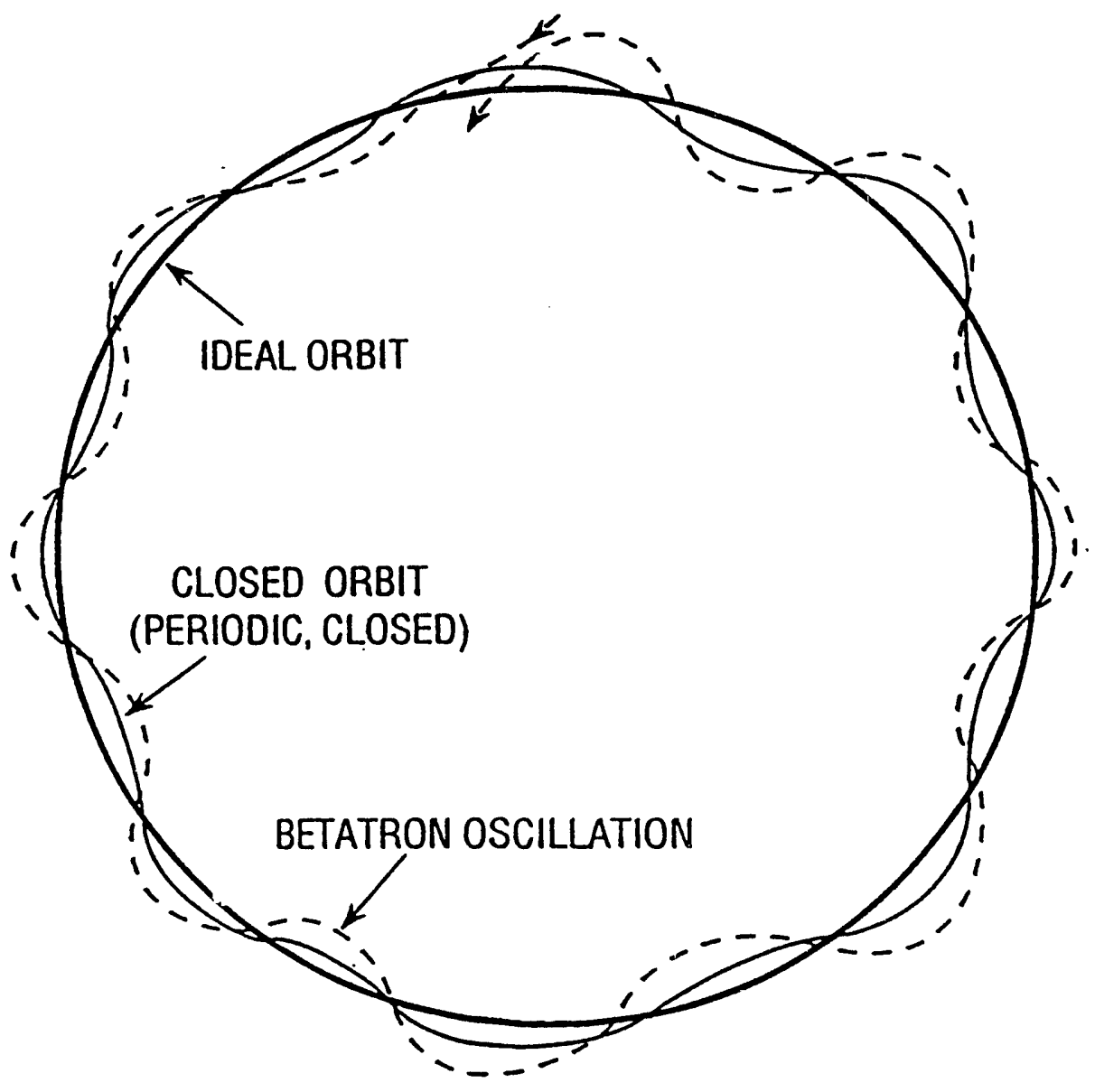

Fig. 2.1 The ideal orbit and closed orbit close after one turn. The betatron oscillation is quasiperiodic and always open.

and the solution for the momentum $\vec{p}$ in the accelerator physics reference frame is

$$
\vec{p}=\left(\begin{array}{ccc}
\cos \theta & 0 & \sin \theta \\
0 & 1 & 0 \\
-\sin \theta & 0 & \cos \theta
\end{array}\right)\left(\vec{P}\left(s_{0}\right)+\int_{s_{0}}^{s} F\left(s^{\prime}\right) \frac{d t}{d s^{\prime}} d s^{\prime}\right) .
$$

Differentiating this with respect to $s$, noting that $d \theta / d s=h$, and using a prime to denote $d / d s$, we obtain

$$
\vec{p}^{\prime}=\vec{F} t^{\prime}+(0, h, 0) \times \vec{p} .
$$


We shall return to this equation below, but first we need some expressions for $d x / d s, d y / d s$, and $d t / d s$. Note that for a particle at position $s$ with coordinates $(x, y)$, the slope of the particle trajectory with respect to the reference trajectory is $p_{x, y} / p_{z}$. Suppose we increment $s$ by a small distance $\Delta s$, and $x$ and $y$ are incremented by $\Delta x$ and $\Delta y$, respectively. Then, because of the curvature of the reference trajectory, the actual distance the particle travels parallel to the reference trajectory is $(1+h x) \Delta s$ to first order in $\Delta s$, hence its horizontal slope is given by $\Delta x /[(1+h x) \Delta s]$, from which we deduce

$$
\frac{p_{x}}{p_{z}}=\frac{x^{\prime}}{1+h x}, \quad x^{\prime}=(1+h x) \frac{p_{x}}{p_{z}}
$$

and, by a similar argument, $y^{\prime}=(1+h x) p_{y} / p_{z}$. The time $\Delta t$ to travel from $s$ to $s+\Delta s$ is given by the distance divided by the velocity, or

$$
\Delta t=\frac{1}{v}\left[(\Delta s)^{2}(1+h x)^{2}+(\Delta x)^{2}+(\Delta y)^{?}\right]^{1 / 2}
$$

and, using the expressions for $x^{\prime}$ and $y^{\prime}$, we deduce that

$$
t^{\prime}=\frac{1+h x}{v}\left[1+\frac{p_{x}^{2}+p_{y}^{2}}{p_{z}^{2}}\right]^{1 / 2}=\frac{1+h x}{v} \frac{p}{p_{z}} .
$$

Using the above results in Eq. (2.5) ( $p_{0}$ being the momentum of the reference particle), we find that

$$
\frac{d}{d s}\left(\frac{p_{x}}{p_{0}}, \frac{p_{y}}{p_{0}}, \frac{p_{z}}{p_{0}}\right)=e\left(\vec{E}+\frac{\vec{v}}{c} \times \vec{B}\right) \frac{1+h x}{v p_{0}} \frac{p}{p_{z}}+h\left(\frac{p_{z}}{p_{0}}, 0,-\frac{p_{x}}{p_{0}}\right) .
$$

Exercise Derive Eqs. (2.2) through (2.9) for yourself, drawing suitable diagrams to illustrate the angles and curvatures involved. Also write out Eq. (2.9) component by component.

The above equations are clearly nonlinear in the derivatives, and $\vec{E}$ and $\vec{B}$ can also depend nonlinearly on $x$ and $y$ (and $s$ ). However, in these notes we shall treat only linear equations of motion. Let us thus neglect all nonlinear terms, e.g. products of derivatives, etc. 
Let us also treat only external magnetic fields, so we put $\vec{E}=0$, and we also put $B_{z}=0$, i.e. we asume the magnetic fields are transverse to the reference trajectory. We also assume that $p_{z}^{2} \gg p_{x, y}^{2}$, which is a very good approximation, so we put $p_{z}=p_{0}=$ constant. After all these simplifying assumptions, we find that

$$
\begin{aligned}
& \frac{d^{2} x}{d s^{2}} \simeq-\frac{e B_{y}}{p_{0}}(1+h x)^{2}+h(1+h x) \\
& \frac{d^{2} y}{d s^{2}} \simeq \frac{e B_{x}}{p_{0}}(1+h x)^{2}
\end{aligned}
$$

which actually still contain some nonlinear terms. It is adequate to treat only the equation for $x$. The right-hand side can be expanded in powers of $x$ and $y$, and at the simplest level the betatron oscillations are described by Hill's equation

$$
\frac{d^{2} x}{d s^{2}}+k(s) x=0
$$

where

$$
k(s+C)=k(s)
$$

where $C$ is the circumference of the closed orbit. The function $k$ is analogous to a spring constant in a harmonic oscillator, and is due mainly to the quadrupole magnets. In more detail, the magnetic field in a quadrupole can be expressed as

$$
\vec{B}_{\text {quad }}=\frac{d B}{d x} x \hat{y}+\frac{d B}{d y} y \hat{x}=\frac{d B}{d x}[x \hat{y}+y \hat{x}] .
$$

We assume the closed orbit is a straight line along the axis of the quadrupole (so $h=0$ ). It is easily verified that $\vec{\nabla} \cdot \vec{B}=0$ and $\vec{\nabla} \times \vec{B}=0$. Then, from Eq. (2.10), we see that

$$
k_{x}=\frac{d B}{d x} \frac{1}{B \rho}, \quad k_{y}=-\frac{d B}{d x} \frac{1}{B \rho} .
$$

The term $B \rho$, usually called B-rho, is an important quantity known as the magnetic rigidity of a particle on the closed orbit, and is given. by

$$
B \rho=p_{0} c / e
$$


so it is proportional to the momentum of a particle on the closed orbit. In Gaussian units, if the magnetic field is measured in Tesla and length in meters,

$$
B \rho \simeq 3.3356 p
$$

where $p$ is measured in $\mathrm{GeV} / \mathrm{c}$. In a magnet with a constant vertical magnetic field $B_{y}=B$, where the reference orbit is a horizontal circular arc, $\rho=h^{-1}$ and it would be the actual radius of the reference orbit. In a real synchrotron, where the closed orbit is not exactly a circle, $B \rho$ is defined by the above equation. The general solution of Eq. (2.11) is

$$
\begin{aligned}
x & =\sqrt{\epsilon \beta(s)} \cos [\psi(s)+\alpha] \\
\psi\left(s_{1} \rightarrow s_{2}\right) & =\int_{s_{1}}^{s_{2}} \frac{d s}{\beta(s)} .
\end{aligned}
$$

The quantity $\epsilon$ is a constant, i.e. independent of $s$, which will be discussed in a subsequent section. It sets an overall scale factor for the magnitude of $x$, whereas $\beta(s)$ gives the position dependence of the amplitude of $x$. The value of $\beta$ is determined by the design of the accelerator lattice (the layout of magnets), whereas the value of $\epsilon$ is determined by other factors. The function $\psi\left(s_{1} \rightarrow s_{2}\right)$ is the phase advance from $s_{1}$ to $s_{2}$, and $\beta(s)$ is called the beta function, or amplitude function (see Fig. 2.2), and one can show that it satisfies

$$
2 \beta \beta^{\prime \prime}-\beta^{2}+4 k \beta^{2}=4,
$$

where a prime denotes the derivative $d / d s$, and, by definition,

$$
\beta(s+C)=\beta(s) .
$$

In addition to $\beta$, there are two other functions $\alpha$ and $\gamma$, and $\{\alpha, \beta, \gamma\}$ are commonly called the Twiss parameters. The definitions of $\alpha$ and $\gamma$ are

$$
\alpha=-\beta^{\prime} / 2, \quad \gamma=\left(1+\alpha^{2}\right) / \beta .
$$


(a)

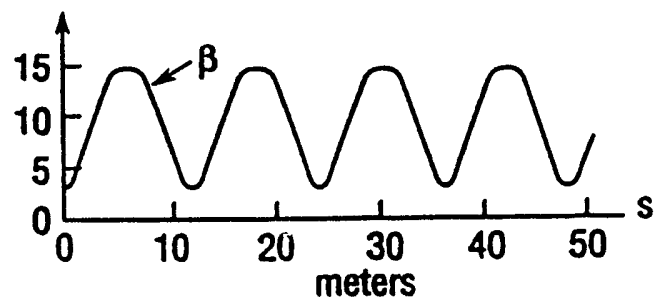

(b)

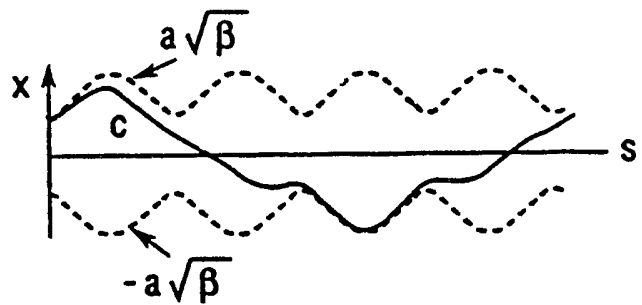

(c)

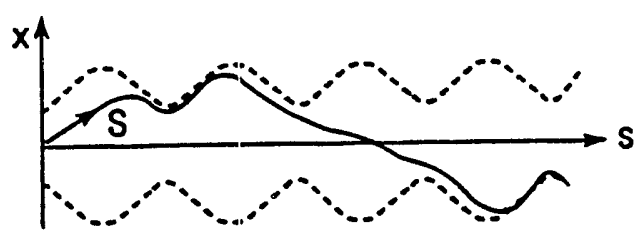

(d)

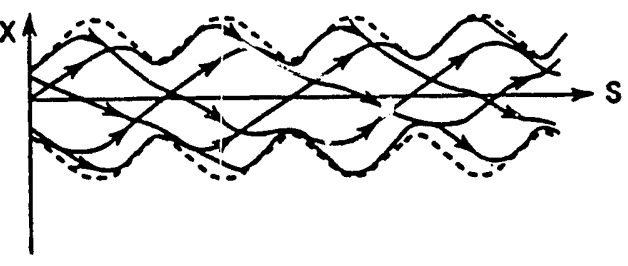

Fig. 2.2 (a) Sketch of a $\beta$ function. (b) Sketch of a cosinelike trajectory (i.e. initial slope $=0$ ). (c) Sketch of a sine-like trajectory (i.e. initial position $=0$ ). (d) Sketch of a trajectory over several turns.

and $\alpha(s+C)=\alpha(s), \gamma(s+C)=\gamma(s)$. In practice, $\beta$ is the most important and most frequently used Twiss parameter (see Fig. 2.3).

Exercise Derive Eq. (2.19) by substitution. of Eq. (2.17) into Hill's equation.

Exercise In a drift space, i.e. a space betwreen magnets, which is free of magnetic fields, there is no focusing, i.e. $k=0$. Show that in a drift space, $\beta$ is given by

$$
\beta(s)=\beta^{*}+\frac{s^{2}}{\beta^{*}},
$$

where $\beta^{*}$ is a constant, and we have conveniently chosen $s=0$ at $\beta=\beta^{*}$. Hence (1) the beta function is parabolic in a drift space, and (2) the curvature of the beta function is inversely proportional to the minimum value $\beta^{*}$. The smaller $\beta^{*}$ is, the more rapidly $\beta(s)$ increases away from $s=0$. 


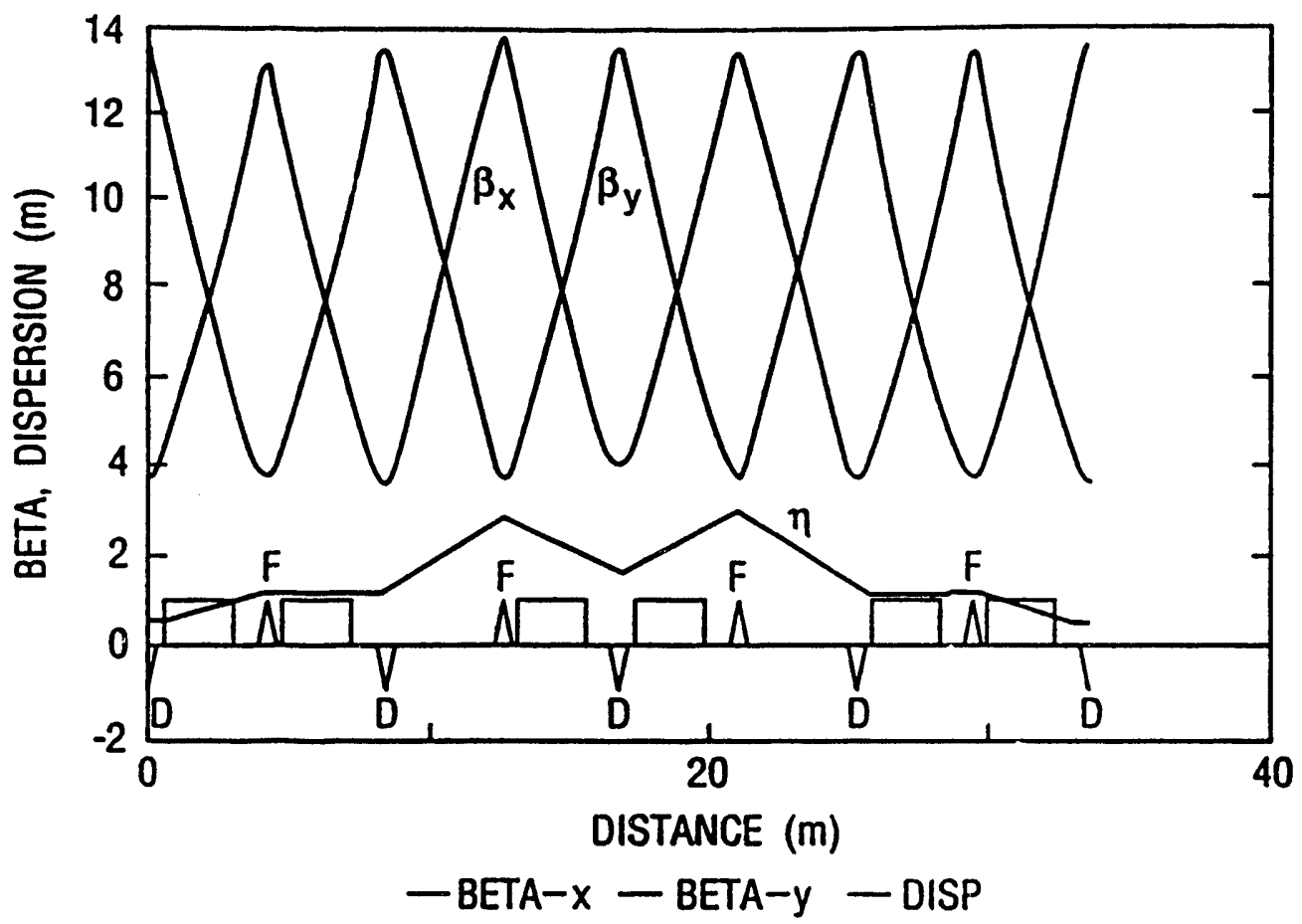

Fig. 2.3 Example of lattice functions for the AGS Booster at BNL. Here $\eta$ denotes the dispersion, to be defined later.

Exercise Prove that $\beta$ is necessarily of one sign throughout the accelerator, i.e. $\beta$ cannot go through zero. Hint: if $\beta=0$ anywhere, then Eq. (2.19) would yield $\beta^{\prime 2}=-4$, which is impossible because $\beta$ is real, by definition. We always take $\beta$ to be positive. Note, however, that $\alpha$ can go through zero, and change sign. This is obvious from the solution for $\beta$ in a drift space, where $\alpha=-s / \beta^{*}$, and changes sign at $s=0$.

Exercise Let us treat an accelerator model known as a weak focusing synchrotron. In this model, the magnetic field is purely transverse $\left(B_{z}=0\right)$, and $B_{x}$ and $B_{y}$ are independent of $s$. The magnetic field consists of a dipole term $B_{y}=B_{0}$ plus a quadrupole term, so

$$
B_{x}=\frac{d B}{d y} y, \quad B_{y}=B_{0}+\frac{d B}{d x} x .
$$


The closed orbit is a horizontal circle $(x=y=0)$. Prove that its radius is $\rho=h^{-1}$. It is conventional to write

$$
B_{x}=-B_{0} n h y, \quad B_{y}=B_{0}(1-n h x),
$$

where $n=-\left(\rho / B_{0}\right) d B / d x$. Prove that Hill's equations for a weak focusing synchrotron are

$$
\frac{d^{2} x}{d s^{2}}=-h^{2}(1-n) x, \quad \frac{d^{2} y}{d s^{2}}=-h^{2} n y .
$$

Deduce that $k_{x}+k_{y}=h^{2}=$ constant. This is a characteristic relation of a weak focusing synchrotron. Deduce that, for stable betatron oscillations in both planes, we must have

$$
0<n<1 \text {. }
$$

Deduce that $\beta_{x}=\rho / \sqrt{1-n}$ and $\beta_{y}=\rho / \sqrt{n}$.

\subsection{Hamiltonian Formulation}

The equation of motion reminds us of simple harmonic motion, except the spring constant is position dependent. Under the Courant-Snyder transformation (see Ref. 3)

$$
\eta=\frac{x}{\sqrt{\beta}}, \quad \phi=\int \frac{d s}{\nu \beta}
$$

it becomes

$$
\frac{d^{2} \eta}{d \phi^{2}}+\nu^{2} \eta=0
$$

where $\nu=(2 \pi)^{-1} \oint d s / \beta$ is the tune of the machine and is a pure number (constant), hence we obtain simple harmonic motion.

Exercise Derive Eq. (2.27). It is simplest to note that $\eta=$ $\sqrt{\epsilon} \cos (\psi+\alpha)$ and $d \psi / d s=\beta^{-1}$, so $d \psi / d \phi=\nu$.

Exercise A widely used approximation in accelerator physics is the smooth approximation, where the focusing and bending are 
assumed to be uniform around the accelerator circumference. In that case, $k$ and $\beta$ are constants. Use Eq. (2.19) to show that, if $\beta^{\prime}=\beta^{\prime \prime}=0$, then $k=\beta^{-2}$. Show also, from the definition of $\nu$, that

$$
\beta \simeq \frac{C}{2 \pi \nu}=\frac{R}{\nu}
$$

where $R \equiv C / 2 \pi$ is the average machine radius. Even in real, i.e. non-smooth, synchrotrons, this generally provides a good relation between the average value of $\beta$, the circumference, and the tune. It is a useful rule of thumb when designing accelerators.

A further transformation,

$$
\eta=\sqrt{2 J / \nu} \cos \lambda, \quad p_{\eta}=d \eta / d \phi=-\sqrt{2 J \nu} \sin \lambda,
$$

makes

$$
H=\frac{p_{\eta}^{2}}{2}+\frac{\nu^{2} \eta^{2}}{2}=\nu J
$$

a constant. Clearly, $H$ is a Hamiltonian for the motion of $\eta$. The quantity $J$ is a constant, known as the action variable, and $\lambda$ is the angle variable. The equations of motion for $J$ and $\lambda$ are

$$
d J / d \phi=0, \quad d \lambda / d \phi=\nu .
$$

Instead of following the particle motion in coordinate space, the corresponding motion can be traced out in phase space (see Fig. 2.4).

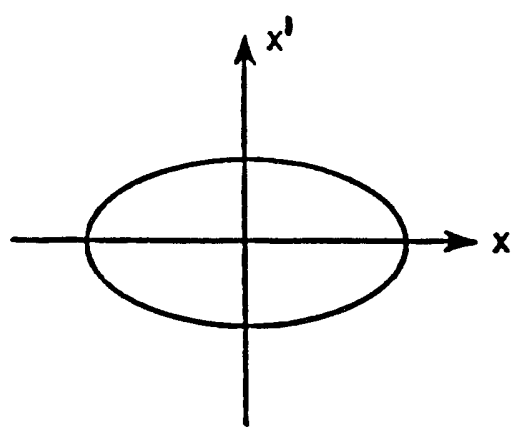

ELLPSE

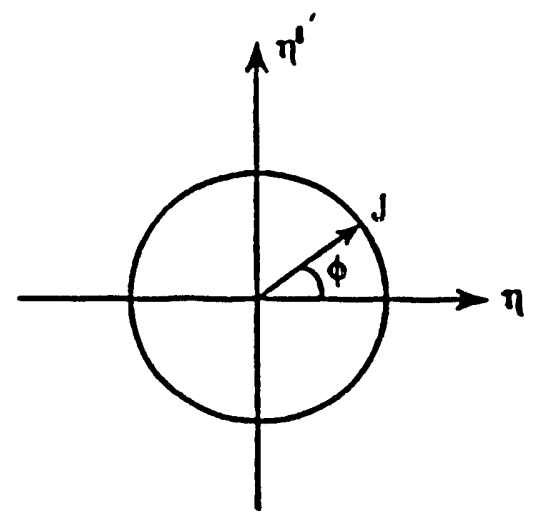

CIRCLE

Fig. 2.4 Sketches of a trajectory as viewed in $\left(x, x^{\prime}\right)$ and $\left(\eta, \eta^{\prime}\right)$ phase space. 
The advantage of the phase-space description, especially with the action-angle variables $(J, \lambda)$, will become apparent when we discuss higher-order terms in the equation of motion. (See also Ref. 4)

\section{Machine Lattice}

\subsection{Comments on Tune Choice}

In general, the motion of a particle will become unstable at a resonance. At a resonance, the particle amplitude will become very large (growing to infinity in principle), and the particle will hit the wall of the vacuum chamber and be lost. A resonance occurs when the condition

$$
m \nu_{x}+n \nu_{y}=k
$$

is satisfied, where $m, n$, and $k$ are positive or negative integers, including zero. The notation $\nu_{x, y}$ denotes the horizontal and vertical betatron tunes. In practice, resonances with $m$ and $n$ both positive actually do not lead to unlimited growth. Only those where $m$ and $n$ have opposite sign (or either $m$ or $n$ is zero) do so.

Strictly speaking, the above condition is very restrictive, because there will be a resonance almost everywhere. We can draw a plot of $\nu_{y}$ vs. $\nu_{x}$, called a tune diagram or tune plane, and in the tune plane, the locations of the resonances will be given by straight lines (see Fig. 3.1). We easily see that the set of all resonances is dense in the plane. In practice, however, it is only necessary to avoid low-order resonances, i.e. those where $|m|+|n|$ is small (the value of small depends on how long the particles are to be stored in the accelerator). The value of $\left(\nu_{x}, \nu_{y}\right)$ in the tune plane is called the working point or operating point.

We therefore see that it is important to choose tunes such that $\nu \neq$ integer, $\nu \neq 1 / 2$ integer, etc. In the subsequent sections, we shall consider the (harmful) consequences of choosing $\nu=$ integer, etc. 


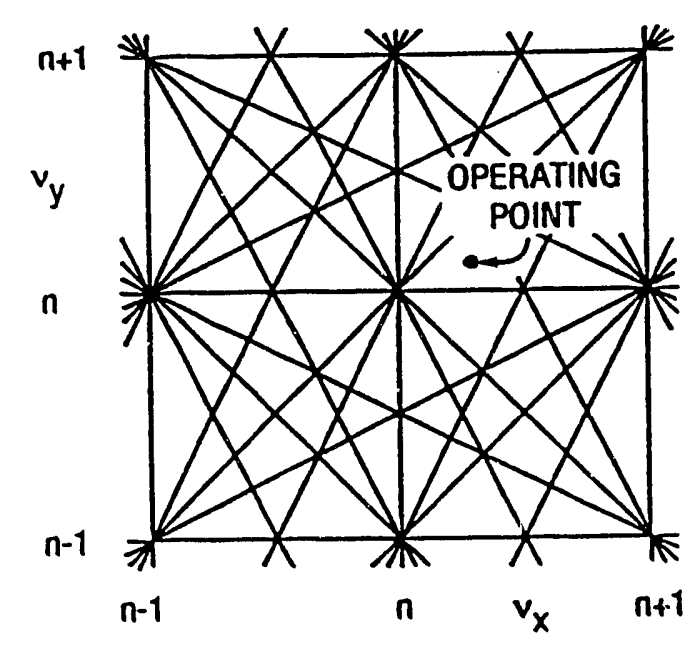

Fig. 3.1 Example of a tune plane with lines denoting various resonances.

\subsection{Orbit Distortion due to Field Error}

In the presence of a field error $\Delta B$ in the dipoles, the actual closed orbit deviates from the ideal orbit,

$$
\frac{d^{2} x}{d s^{2}}+k(s) x=-\frac{\Delta B}{B \rho} \equiv F(s) .
$$

Since the field error is in the dipoles, $\Delta B$ depends on $s$, but not on $x$. The Courant-Snyder transformation yields

$$
\frac{d^{2} \eta}{d \phi^{2}}+\nu^{2} \eta=\nu^{2} \beta^{3 / 2} F(\phi) .
$$

The Green function method gives the periodic solution

$$
\eta(\phi)=\frac{\nu}{2 \sin (\pi \nu)} \int_{\phi}^{\phi+2 \pi} \beta^{3 / 2} F\left(\phi^{\prime}\right) \cos \left[\nu\left(\pi+\phi-\phi^{\prime}\right)\right] d \phi^{\prime}
$$

as long as $\nu \neq$ integer. We see that if $\nu=$ integer, then the closed orbit $\rightarrow \infty$, i.e. even a small field error $\Delta B$ will cause a large closed orbit distortion. Since small errors $\Delta B$ are always present, this makes it undesirable to choose a tune close to an integer. 
Exercise Prove Eq. (3.4). Hint: first derive the Green function $G\left(\phi, \phi^{\prime}\right)$ which satisfies

$$
\frac{d^{2} G}{d \phi^{2}}+\nu^{2} G=\delta\left(\phi-\phi^{\prime}\right)
$$

with the condition $G\left(\phi=0, \phi^{\prime}\right)=G\left(\phi=2 \pi, \phi^{\prime}\right)$, i.e. $G$ is periodic in $\phi$ (and $d G / d \phi$ is also periodic). Then use

$$
\eta(\phi)=\int_{\phi}^{\phi+2 \pi} \nu^{2} \beta^{3 / 2} G\left(\phi, \phi^{\prime}\right) F\left(\phi^{\prime}\right) d \phi^{\prime} .
$$

Note that the condition $\sin (\pi \nu) \neq 0$ will be required to obtain a well-defined Green function.

\subsection{Amplitude Function Distortion due to Gradient Error}

In the above case, the field error $\Delta B$ was a function of $s$ but not $x$. Suppose now there is a gradient error $\Delta k$ in the quadrupoles. Then

$$
\frac{d^{2}}{d \phi^{2}} \frac{\Delta \beta}{\beta}+4 \nu^{2} \frac{\Delta \beta}{\beta}=-2 \nu^{2} \beta^{2} \Delta k(\phi),
$$

and, provided $\nu \neq 1 / 2$ integer, the Green function technique yields

$$
\frac{\Delta \beta}{\beta}(\phi)=-\frac{\nu}{\sin (2 \pi \nu)} \int_{\phi}^{\phi+2 \pi} \beta^{2} \Delta k \cos \left[2 \nu\left(\pi+\phi-\phi^{\prime}\right)\right] d \phi^{\prime} .
$$

Exercise Derive Eq. (3.7). Hint: first substitute

$$
\beta \rightarrow \beta\left(1+\frac{\Delta \beta}{\beta}\right), \quad k \rightarrow k+\Delta k
$$

into Eq. (2.19) to obtain, to first order in small quantities,

$$
\left(\frac{\Delta \beta}{\beta}\right)^{\prime \prime}+\frac{\beta^{\prime}}{\beta}\left(\frac{\Delta \beta}{\beta}\right)^{\prime}+\frac{4}{\beta^{2}} \frac{\Delta \beta}{\beta}+2 \Delta k=0,
$$

where a prime denotes the derivative $d / d s$. Then use $d s=\nu \beta d \phi$ to convert the derivatives to $d / d \phi$. Eq. (3.7) should follow immediately. 
Exercise Prove Eq. (3.8). In fact, the result can almost be copied from Eq. (3.4).

We see that this time the closed orbit is not affected, but rather the amplitude of the betatron oscillations is perturbed. At a halfinteger tune, the amplitude grows very large, resulting in unstable particle motion and beam loss.

\subsection{Momentum Effects}

\subsubsection{Dispersion}

For a particle with momentum $p=p_{0}+\Delta p$, the equation of motion becomes

$$
\frac{d^{2} x}{d s^{2}}+k(s) x=\frac{1}{\rho} \frac{\Delta p}{p_{0}} .
$$

This is a linear differential equation with a driving term on the right, so we can divide the solution into a betatron part (which satisfies the homogenous equation) and a driven part, proportional to $\Delta p / p_{0}$. Let us write

$$
x=x_{p}+x_{\beta}=D(s) \frac{\Delta p}{p_{0}}+x_{\beta},
$$

i.e. $x_{\beta}$ is the betatron oscillation part, and $x_{p}$ is proportional to the momentum offset. Then

$$
D^{\prime \prime}+k D=\frac{1}{\rho} \text {. }
$$

We further demand that $D$ be periodic, i.e. $D(s+C)=D(s)$. Then the solution is

$$
D(\phi)=\frac{\nu \sqrt{\beta}}{2 \sin (\pi \nu)} \int_{\phi}^{\phi+2 \pi} \frac{\beta^{3 / 2}}{\rho} \cos \left[\nu\left(\pi+\phi-\phi^{\prime}\right)\right] d \phi^{\prime}
$$

provided $\nu \neq$ integer. The derivation is again similar to that of Eq. (3.4), since $\rho$ does not depend on $x$. Here $D$ is called the dispersion function of the machine. In other words, a particle with a momentum offset $\Delta p / p_{0}$ follows a closed orbit which is given by 
$D \Delta p / p_{0}$ relative to the closed orbit of an ideal particle, and it executes betatron oscillations around this new closed orbit. Neglecting the betatron oscillations, we see that the momentum offset changes the path length or circumference of the closed orbit. The relative path increment is called the momentum compaction factor $\alpha$,

$$
\alpha=\frac{\Delta R / R}{\Delta p / p_{0}}
$$

and one can show that

$$
\alpha=\frac{1}{2 \pi R} \oint \frac{D}{\rho} d s,
$$

where $R=C / 2 \pi$ is the average machine radius.

Exercise We can use the smooth approximation to obtain a useful rule-of-thumb estimate of the value of the dispersion. Assume that $\beta$ and $\rho$ are constants, so $\rho=R$ and $\beta=R / \nu$, independent of $\phi$. Then evaluate the integral in Eq. (3.14) to show that

$$
D \simeq \beta / \nu \text {. }
$$

This result is again approximately valid in real synchrotrons.

Exercise Derive Eq. (3.16). Hint: draw a diagram of an arc of radius $\rho$ and arc length $\Delta s$. The angle subtended at the center of curvature is $\Delta \theta=\Delta s / \rho$. For infinitesimal $\Delta \theta$, the dispersion $D$ will be nearly constant, so the radius of the off-momentum closed orbit will be $\rho+\left(D \Delta p / p_{0}\right)$, and thus the path difference will be $\left(D \Delta p / p_{0}\right) \Delta \theta$, or $(D / \rho) \Delta p / p_{0} \Delta s$. Integrating around the circumference, the increase in circumference is

$$
\Delta C=2 \pi \Delta R=\frac{\Delta p}{p_{0}} \oint \frac{D}{\rho} d s,
$$

and the rest of the derivation follows easily. 
Exercise Using the smooth approximation, where $D \simeq \beta / \nu$, use Eq. (3.16) to show that

$$
\alpha \simeq \nu^{-2}
$$

This shows that $\alpha$ is usually a small number, since usually $\nu \gg 1$.

\subsubsection{Chromaticity}

The tune is also changed for a particle with a momentum offset. The tune change per $\Delta p / p_{0}$ is called the chromaticity $\xi$,

$$
\xi \equiv \frac{\Delta \nu}{\Delta p / p_{0}}=-\frac{1}{2 \pi} \oint \frac{\Delta \beta / \beta}{\Delta p / p_{0}} \frac{d s}{\beta}
$$

which produces a tune spread for a beam with a momentum spread.

Exercise Using the result $\nu=(2 \pi)^{-1} \oint d s / \beta$ and $\beta \rightarrow \beta+\Delta \beta$, show that

$$
\Delta \nu=-\frac{1}{2 \pi} \oint \frac{\Delta \beta}{\beta} \frac{d s}{\beta},
$$

from which Eq. (3.20) follows immediately.

Exercise In a quadrupole, the dependence of $k$ on $\Delta p / p_{0}$ is given by

$$
\begin{aligned}
k & =\frac{d B / d x}{B \rho} \propto \frac{d B / d x}{p}=\frac{d B / d x}{p_{0}+\Delta p} \\
& \rightarrow \frac{k_{0}}{1+\left(\Delta p / p_{0}\right)} \simeq k_{0}\left(1-\frac{\Delta p}{p_{0}}\right)
\end{aligned}
$$

hence $\Delta k=-k \Delta p / p_{0}$. Use this result in Eq. (3.8) and use Eq. (3.8) to substitute for $\Delta \beta / \beta$ in Eq. (3.20). By interchanging the orders of integration, or otherwise, show that the resulting double integral can be simplified to

$$
\xi=-\frac{1}{2 \pi} \oint k \beta d s .
$$


This elegant and simple result gives the so-called natural chromaticity of an accelerator, i.e. the chromaticity due to the quadrupoles. Sextupole magnets can also contribute to the chromaticity, but we shall not treat them here.

Exercise Eq. (3.23) can be used to perform some useful numerical estimates. Let us employ the smooth approximation, where we assume that $k$ and $\beta$ are constant around the ring circumference. Using Eq. (2.19), show that $k=\beta^{-2}$. Use this and Eq. (3.23) to show that, in the smooth approximation,

$$
\xi \simeq-\frac{1}{2 \pi} \oint \frac{d s}{\beta}=-\nu
$$

This result is approximately true even in non-smooth accelerators. It states that the natural chromaticity is roughly the negative of the tune.

\section{Representation of a Particle Beam}

\subsection{Introduction}

Up to now we have treated an individual particle and properties of the accelerator lattice that relate to an individual particle. In the next section we shall treat a beam of particles, i.e. an ensemble of several particles. Hence now is the time to review the particle trajectory and machine lattice topics described above. Remember that the betatron oscillations create an amplitude $x_{\beta}=\sqrt{\epsilon \beta}$ which determines the profile of the beam. Usually the ideal design orbit follows the center of the vacuum chamber, but the actual closed orbit is not on the ideal design orbit. There will be various obstructions in the vacuum chamber, e.g. pickup electrodes to monitor the beam position, a septum for injecting/extracting particles into/out of the accelerator, etc., and so the beam must stay clear of them, as sketched in Fig. 4.1. Hence we design an accelerator, i.e. the function $\beta$, with such goals in mind. We discuss $\epsilon$ (which is a constant independent of $s$ ) below. 


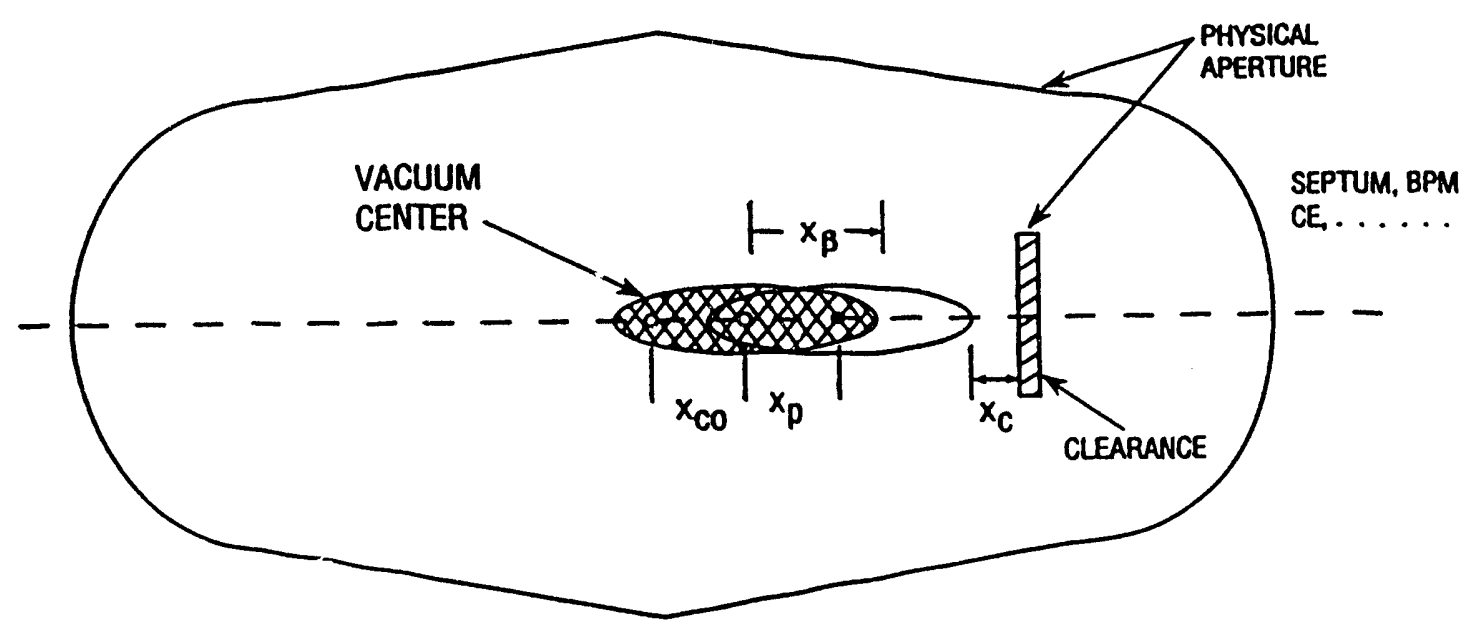

$$
\begin{aligned}
\text { BEAM SIZE } & x_{B}=x_{B}+x_{p} \\
\text { BEAM EXTENT } & x_{E}=x_{C O}+x_{B} \\
\text { APERTURE } & x_{A}=x_{E}+x_{C} \\
\text { BEAM STAY CLEAR } & x_{B S C}=x_{C O}+(6-8) x_{\beta} \\
\text { PHYSICAL APERTURE } & x_{P A}=\text { MAX }\left(x_{A}, x_{B S C}, x_{\text {WAUL }}\right)
\end{aligned}
$$

Fig. 4.1 Sketch of a beam in a vacuum chamber.

The shape of the beta function is determined by the accelerator lattice of dipole and quadrupole magnets. In addition, the tune $\nu$ of the betatron oscillations is also determined by $\beta$, being basically the average of $\beta^{-1}$. We have seen above that the tunes $\left(\nu_{x}, \nu_{y}\right)$ determine a point in the tune plane, and there are many resonances in this plane. Since the particle motion can become unstable at a resonance, it is important to choose the tune carefully to avoid harmful resonances. We gave examples of driving terms that could excite resonances at $\nu=$ integer and $\nu=1 / 2$ integer above, e.g. dipole field errors (closed orbit distortions) or gradient errors. We also showed, at the end, that a beam of particles with a distribution of values $\Delta p / p_{0}$, i.e. a nonzero momentum spread, would also have a distribution of tunes, proportional to the chromaticity, so that the operating point in the tune plane would be spread over many values. There are many other sources of tune spread that we shall not treat here. 
We also briefly discussed the coordinate and phase-space descriptions of the particle motion, leading to the powerful concept of action-angle variables. We did not actually make use of actionangle variables above, but they are useful, e.g. in the treatment of nonlinear dynamics.

For physics research we need high beam intensity as well as high energy. Particle beams are produced with finite extent in the sixdimensional phase space. The aim is to keep the extent of the beam distribution small and constant, i.e. not to blow the beam up, through acceleration and also during transfer between accelerators. For real beams the distribution can take all shapes, but it is convenient to use idealized mathematical models to represent them. The most popular two are elliptical and Gaussian.

\subsection{Elliptical Distribution}

- Uniform distribution in $\left(x, x^{\prime}\right)$ phase-space

- Circular (or elliptical) boundary

- One-dimensional projection onto $x$-axis is elliptical

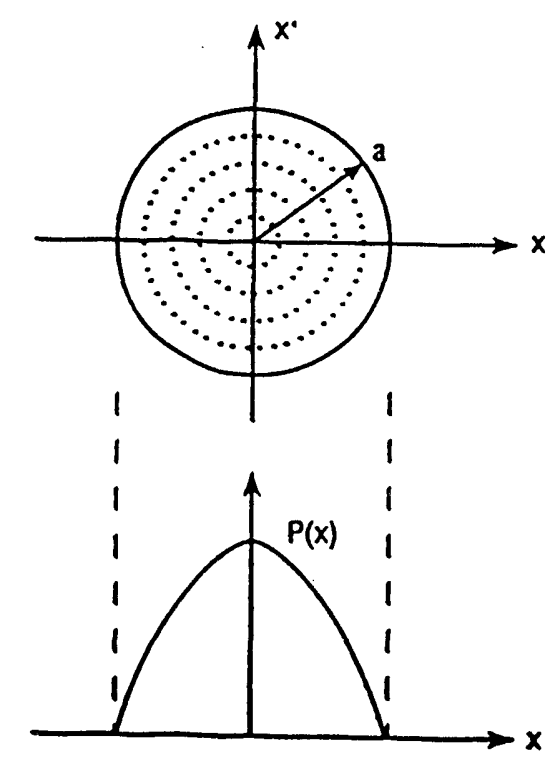

$$
N=\int \rho\left(x, x^{\prime}\right) d x d x^{\prime}=\rho \pi a^{2}
$$

where $\rho=$ constant. Hence $a=\sqrt{N /(\rho \pi)}$. Then

$$
p(x)=2 \rho \sqrt{a^{2}-x^{2}}=\frac{2 N}{\pi a}\left(1-\frac{x^{2}}{a^{2}}\right)^{1 / 2}
$$

Exercise Derive Eq. (4.2). 


\subsection{Gaussian Distribution}

- Gaussian distribution in $\left(x, x^{\prime}\right)$ phase-space

- Constant density contour is elliptical

$-x$ projection is Gaussian
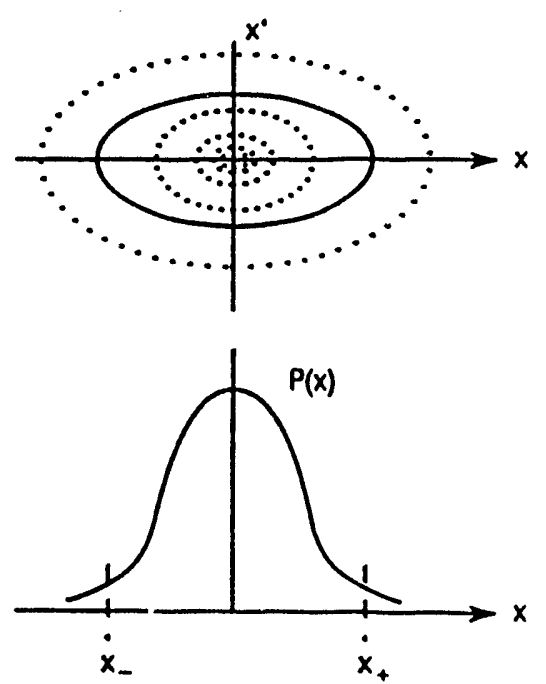

Assume that the constant density contour is elliptical and can be represented by

$$
\epsilon=\frac{x^{2}+\left(\alpha x+\beta x^{\prime}\right)^{2}}{\beta}=\gamma x^{2}+2 \alpha x x^{\prime}+\beta x^{\prime 2}
$$

where $\alpha, \beta$, and $\gamma$ are the Twiss parameters [see Eq. (2.21)]. Note that $\beta \gamma-\alpha^{2}=1$. Assume further that the density distribution is Gaussian,

$$
\begin{gathered}
\rho\left(x, x^{\prime}\right)=K \exp \left[-C\left(\gamma x^{2}+2 \alpha x x^{\prime}+\beta x^{\prime 2}\right)\right], \\
\int \rho\left(x, x^{\prime}\right) d x d x^{\prime}=N, \quad \Rightarrow C=\frac{K \pi}{N} .
\end{gathered}
$$

The $x$-projection (spatial distribution) is

$$
p(x)=\int_{-\infty}^{\infty} \rho\left(x, x^{\prime}\right) d x^{\prime}=\left(\frac{K}{\beta}\right)^{1 / 2} \exp \left[-\frac{K \pi}{N \beta} x^{2}\right] .
$$

Exercise Derive Eq. (4.6).

Once we have established the spatial distribution as Gaussian, there are two important relations to know. First is the point where the beam intensity is $e^{-n}$ of its peak value at the center,

$$
x_{ \pm n}= \pm\left(n \frac{N \beta}{K \pi}\right)^{1 / 2} \text {. }
$$


Second is the fraction of the beam contained within this boundary. In 2-D phase-space this is

$$
f=\int_{x_{-}}^{x_{+}} \int_{x_{-}^{\prime}}^{x_{+}^{\prime}} \rho\left(x, x^{\prime}\right) d x d x^{\prime}=1-e^{-n} .
$$

The fraction of the beam contained within this boundary in spatial projection is

$$
F=\int_{-\infty}^{\infty} d x^{\prime} \int_{x_{-}}^{x_{+}} \rho\left(x, x^{\prime}\right) d x=\operatorname{erf}(\sqrt{2 n})=\frac{1}{\sqrt{2 \pi}} \int_{-\sqrt{2 n}}^{\sqrt{2 n}} e^{-t^{2} / 2} d t .
$$

Exercise Prcve Eqs. (4.8) and (4.9).

The useful numerical values can be summarized in Table 1.

Table 1

\begin{tabular}{llll}
\hline$n$ & $e^{-n}$ & $F(1-D)$ & $f(2-D)$ \\
\hline 1 & 0.3679 & 0.8426 & 0.6321 \\
2 & 0.1353 & 0.9544 & 0.8647 \\
3 & 0.0498 & 0.9858 & 0.9502 \\
4 & 0.0183 & 0.9954 & 0.9817 \\
\hline
\end{tabular}

Question How much beam is left if beam scrapers are put at $x_{ \pm 2}$ at (1) transport line, (2) storage ring?

Exercise What are the corresponding $F$ and $f$ at FWHM?

Exercise Derive equivalent expressions for 3-D, 4-D, 5-D, and 6-D distributions.

\subsection{Emittance and Admittance}

See also Ref. 5 for more details. The emittance $\epsilon$ is the 2dimensional phase-space area occupied by a trajectory,

$$
\text { Area }=\pi \epsilon=\gamma x^{2}+2 \alpha x x^{\prime}+\beta x^{\prime 2} .
$$


This $\epsilon$ is the same as the one we chose to represent the amplitude of a b statron oscillation,

$$
x=\sqrt{\epsilon \beta} \cos (\psi+\delta) .
$$

In an accelerator, the smallest limiting physical aperture, say $x=$ $a$, occurs at the point of maximum $\beta$. At that point $\alpha=x^{\prime}=0$, and $\gamma=\beta^{-1}$. The corresponding emittance at that point is called the admittance of the machine

$$
\text { admittance }=\epsilon_{\min }=a^{2} / \beta_{\max } .
$$

Exercise Show that $\beta$ has dimensions of length, say meters, $\alpha$ is dimensionless, and $\gamma$ has dimensions of inverse length. Show also that $\epsilon$ has dimensions of length. The units of emittance, and admittance, are meter-radians. Frequently one uses a smaller unit, such as millimeter-milliradians $\left(=10^{-6}\right.$ meter-radian $)$.

\subsection{Emittance of a Beam}

The above use of emittance referred to the betatron oscillation of an individual particle. It gave a measure of the amplitude of the betatron oscillation. In practice, however, the term emittance is normally used to refer, not to an individual particle, but to a beam. To avoid confusion between different usages of "emittance," it is preferable to use the concept of action (see action-angle variables above) to refer to an individual particle.

Exercise Show, using the Courant-Snyder transformation $\eta=$ $x / \sqrt{\beta}$, etc., that $\epsilon=2 J / \nu$, so that the betatron oscillation amplitude of an individual particle could be characterized by $J$ rather than $\epsilon$. The use of action is universal when dealing with nonlinear terms in the equation of motion. Note, however, that for linear dynamics the use of the notation $x=\sqrt{\epsilon \beta} \cos (\ldots)$ is widespread, with $\epsilon$ being called the emittance. 
Let us now therefore consider the use of the term emittance as it applies to a beam. For a finite beam with uniform charge distribution in the phase space, the emittance is a well-defined quantity. It refers to the phase-space area enclosed by the beam, i.e. the largest amplitude of all the particle oscillations. For the Gaussian distribution we discussed above, the emittance of the beam has to be related to the fraction of particles included. This is because a Gaussian distribution has tails that stretch to $x \rightarrow \pm \infty$. Since a real beam has only a finite number of particles, we must remember that the use of a Gaussian is only a mathematical idealization, valid for not too large amplitudes. Typically a Gaussian beam is characterized by a parameter called the standard deviation $\sigma$, or variance $\sigma^{2}$,

$$
p(x)=\frac{1}{\sqrt{2 \pi} \sigma} e^{-x^{2} / 2 \sigma^{2}} .
$$

At a point in the accelerator of maximum $\beta$, we have $\alpha=x^{\prime}=0$ and so $\epsilon=x^{2} / \beta$. Let us define

$$
\epsilon_{\mathrm{rms}}=\sigma^{2} / \beta
$$

at this point. Note that $\epsilon_{\text {rms }}$ will not equal $\sigma^{2} / \beta$ at other points in the ring because $\beta$ will change with arc length $s$, whereas $\epsilon_{\text {rms }}$ will not. Then, with an obvious notation,

$$
\epsilon_{95 \%}=x_{95 \%}^{2} / \beta \text {. }
$$

From Table 1, $\epsilon_{95 \%}$ corresponds to $n=3$, hence

$$
\begin{aligned}
e^{-x^{2} / 2 \sigma^{2}} & =e^{-3}, \Rightarrow x_{95 \%}=\sqrt{6} \sigma \\
\epsilon_{95 \%} & =x_{95 \%}^{2} / \beta=6 \sigma^{2} / \beta=6 \epsilon_{\mathrm{rms}} .
\end{aligned}
$$

Exercise Prove that $\epsilon_{86 \%}=4 \epsilon_{\mathrm{rms}}$ and $\epsilon_{99 \%}=9.2 \epsilon_{\mathrm{rms}}$.

In general, when accelerator physicists speak of a certain emittance, say $1 \mathrm{~mm}$-mrad, they are referring to a beam, not a particle, and are using a Gaussian model. Note, however, that it is 
not standard to use the term emittance to refer to $\epsilon_{\mathrm{rms}}$. In some laboratories, the emittance means $\epsilon_{95 \%}$. Hence the reader should check the conventions used by each author. (See also the section below on normalized emittance.)

\subsection{Matching between Two Optical Channels}

It is usually too difficult to accelerate a beam to the final desired energy using only one accelerator. It is necessary to move a beam through stages of accelerators or transport lines, and in general they will have different optical parameters. Then the matching between them is very important, otherwise the emittance will blow up and cause phase-space dilution. The details are summarized in Fig. 4.2.

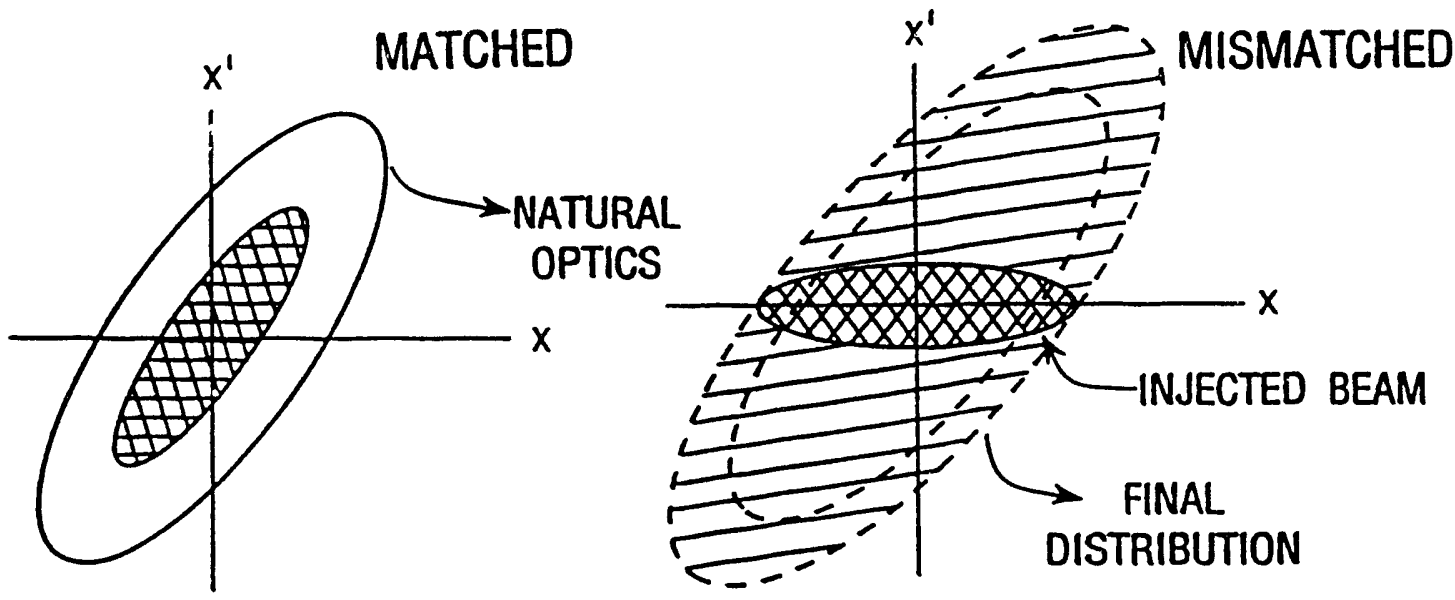

Fig. 4.2 Matching of a beam into a synchrotron. If the contours of the beam follow those of the machine, we say that the beam is matched to the synchrotron. If a beam is mismatched, its emittance will increase, as sketched above.

In phase space, a particle trajectory such as a betatron oscillation will trace out a contour given by $\epsilon=\gamma x^{2}+2 \alpha x x^{\prime}+\beta x^{\prime 2}$, i.e. an ellipse. Thus the contours in the phase space will be ellipses characterized by different values of $\epsilon$. If a beam is injected into the accelerator, and its shape (in phase space) already follows the contours, we say the beam is matched to the optics of the ma- 
chine. In that case, the phase-space shape of the beam will not be changed by the subsequent motion of the beam in the accelerator. This is because, as the particles follow their individual betatron oscillations, they will not enter regions of phase space that were not already occupied by the injected beam distribution. This can be seen from Fig. 4.2. In particular, the r.m.s. emittance of the beam will not increase.

Now suppose, however, that the shape of the injected beam does not follow the phase-space contours of the accelerator, as in the second case shown. In that case, the particles will again trace out their individual betatron oscillations, and will eventually fill out the whole phase-space region of all the values of $\epsilon$ occupied by the injected beam distribution. We see from Fig. 4.2 that this means the beam will now fill out regions of phase space that were not occupied by the injected beam distribution. The phase-space area occupied by the beam will therefore increase or, equivalently, the r.m.s. emittance of the beam will increase: the beam will blow up.

\subsection{Liouville's Theorem}

In practice, we usually wish to create a beam with as small an emittance as possible. In proton or heavy ion accelerators, the phase-space distribution of the beam is determined by the proton or ion source. It cannot be changed by an accelerator lattice described by a Hamiltonian. This is a consequence of Liouville's theorem. This theorem states that, if we have a distribution of points in phase space, described by a density function $\rho$, say $\rho\left(x, x^{\prime}\right)$ for a two-dimensional phase space, and the motion of the particles in that phase space is determined by a Hamiltonian, then the phasespace density will be constant in time, i.e. $d \rho / d t=0$. Hence, if we follow a phase-space volume element $d x d x^{\prime} d y d y^{\prime} \ldots$, the volume element will move through the phase space but the density $\rho$ in that volume element will not change. We can picture the beam as an incompressible fluid moving through the phase space. This hydrodynamic viewpoint is indeed used in the accelerator physics 
literature, but is beyond the scope of these notes. In particular, therefore, the emittance of a beam will not be reduced by motion through the machine lattice. We noted this above when we said $x \propto \sqrt{\epsilon \beta}$ and stated that only $\beta$, but not $\epsilon$, depended on $s$.

The above statements imply that the emittance of a beam is determined at the particle source and cannot be reduced by an accelerator lattice. There are several caveats to this, however. The emittance can be changed through the operation of non-Hamiltonian processes. Liouville's theorem applies only if the motion in the phase space is described by a Hamiltonian. Examples of processes that are not Hamiltonian occurring in an accelerator are residual gas scattering (with gas molecules or ions in the beam pipe), space-charge forces (mutual repulsion of the particles because they have the same sign of charge), power supply noises (leading to random fluctuations in the magnetic fields), etc. Another example is the situation in high energy electron and positron synchrotrons and storage rings, where the particles emit synchrotron radiation. This leads to stochastic fluctuations and radiation damping. The above concepts are beyond the scope of these notes, but we note that they cause electron and positron beams to achieve an equilibrium emittance that is actually independent of their initial emittance. Thus Liouville's theorem does not apply to high energy beams of electrons and positrons in synchrotrons.

Returning to the discussion of matching a beam to an accelerator, we saw that the emittance could increase during the transfer of particles from one accelerator to another, if the beam exiting the first one was not matched to the phase-space contours of the second. In this case, the motion in each accelerator is described by a Hamiltonian, but the Hamiltonians of the two accelerators are different, and so the emittance can change - in particular it may increase - during the transfer. It will not decrease, however, as can be seen from Fig. 4.2. A matched beam can have the same emittance as its initial value, but not a smaller emittance. 


\subsection{Normalized Emittance}

We said above that the emittance has dimensions of meterradians, being basically the product of $x$ and $x^{\prime}$. We also noted, via Liouville's theorem, that the emittance was constant in a synchrotron, i.e. independent of the arc length $s$. However, the emittance, as defined above, is constant only if the energy of the beam does not change. In a real synchrotron, a beam of particles is usually injected into the synchrotron at low energy, and the beam energy is then increased up to a higher final energy. The magnetic fields in the synchrotron magnets, e.g. the dipoles and quadrupoles, have to be increased in proportion to the beam momentum to maintain the shape of the closed orbit and the focusing of the betatron oscillations. The change of energy takes place adiabatically, i.e. the energy increase per revolution around the synchrotron is small, so that the change in the magnetic fields is also adiabatic, and thus the parameters of the phase space, e.g. the Twiss parameters, do not change rapidly during acceleration. Under these conditions, the emittance, as defined above, does not remain constant, but there is a quantity, called the normalized emittance, which does remain constant.

The normalized emittance is related to the above geometric emittance via

$$
\epsilon_{\text {normalized }}=\frac{p_{0}}{m c} \epsilon=\beta_{0} \gamma_{0} \epsilon .
$$

It also has units of meter-radians. Here $p_{0}$ is the momentum on the design orbit, and $\beta_{0}$ and $\gamma_{0}$ are not Twiss parameters, but rather $p_{0}=m v_{0} \gamma_{0}$ and

$$
\beta_{0}=\frac{v_{0}}{c}, \quad \gamma_{0}=\frac{1}{\sqrt{1-\beta_{0}^{2}}},
$$

where $m$ is the particle rest mass and $v_{0}$ is its speed.

When accelerator physicists speak of the emittance of a particle beam of protons or heavy ions, they almost always refer to the normalized emittance. This is not true for electron or positron beams, where emittance usually means the geometric emittance (because there is no point in introducing a normalized emittance). 
The normalized emittance is a constant under acceleration because it is an adiabatic invariant. It is a theorem that when the motion in a phase space is described by a Hamiltonian, the action variable of a trajectory is not merely a constant, but also an adiabatic invariant, i.e. if the Hamiltonian depends on some parameter $\tau$ which changes slowly, then the value of the action will not change as a function of $\tau$. It is beyond the scope of these notes to give a full derivation, but the action that remains invariant is $p_{0} \epsilon$, and it has dimensions of length $\times$ momentum. (We have previously seen that the emittance is proportional to the action for a betatron oscillation.) Up to now, we have taken the phase-space variables to be $\left(x, x^{\prime}\right)$, where $x^{\prime}=d x / d s$. However, the momentum that is canonically conjugate to $x$ is $p_{x}$, where $p_{x}=p_{0} x^{\prime}$. The emittance in the $\left(x, p_{x}\right)$ phase space remains constant during adiabatic changes of the Hamiltonian, i.e. a slow increase of $p_{0}$. Up to now, we have always assumed $p_{0}$ to be constant, and have ignored it. We see that the definition of the normalized emittance is deliberately chosen so that it will remain constant during acceleration to high energy.

\section{Longitudinal Motion - Synchrotron Oscillations}

\subsection{Equations of Motion}

For this topic, see also Ref. 7. In a synchrotron, radio-frequency longitudinal electric fields are created locally across rf cavities to provide an accelerating field to the beam. Typically, the rf frequency is not identical to the revolution frequency. The ratio between them is called the harmonic number $h$

$$
h=\omega_{R F} / \omega_{\text {rev }},
$$

and is an integer. In that case, the circumference of the machine will be divided into $h$ sections. Within each section a stable region will be created and populated by particles. 
The voltage across the accelerating gap is

$$
V(t)=\bar{V} \sin \phi(t) .
$$

Assuming the synchronous particle (s.p.) arrives at the gap at phase $\phi_{0}$, then the energy gain and the increase in the guide field should satisfy

$$
\Delta E_{0}=e \bar{V} \sin \phi_{0}=2 \pi e R_{0}^{2} \frac{d B}{d t} .
$$

See Fig. 5.1.

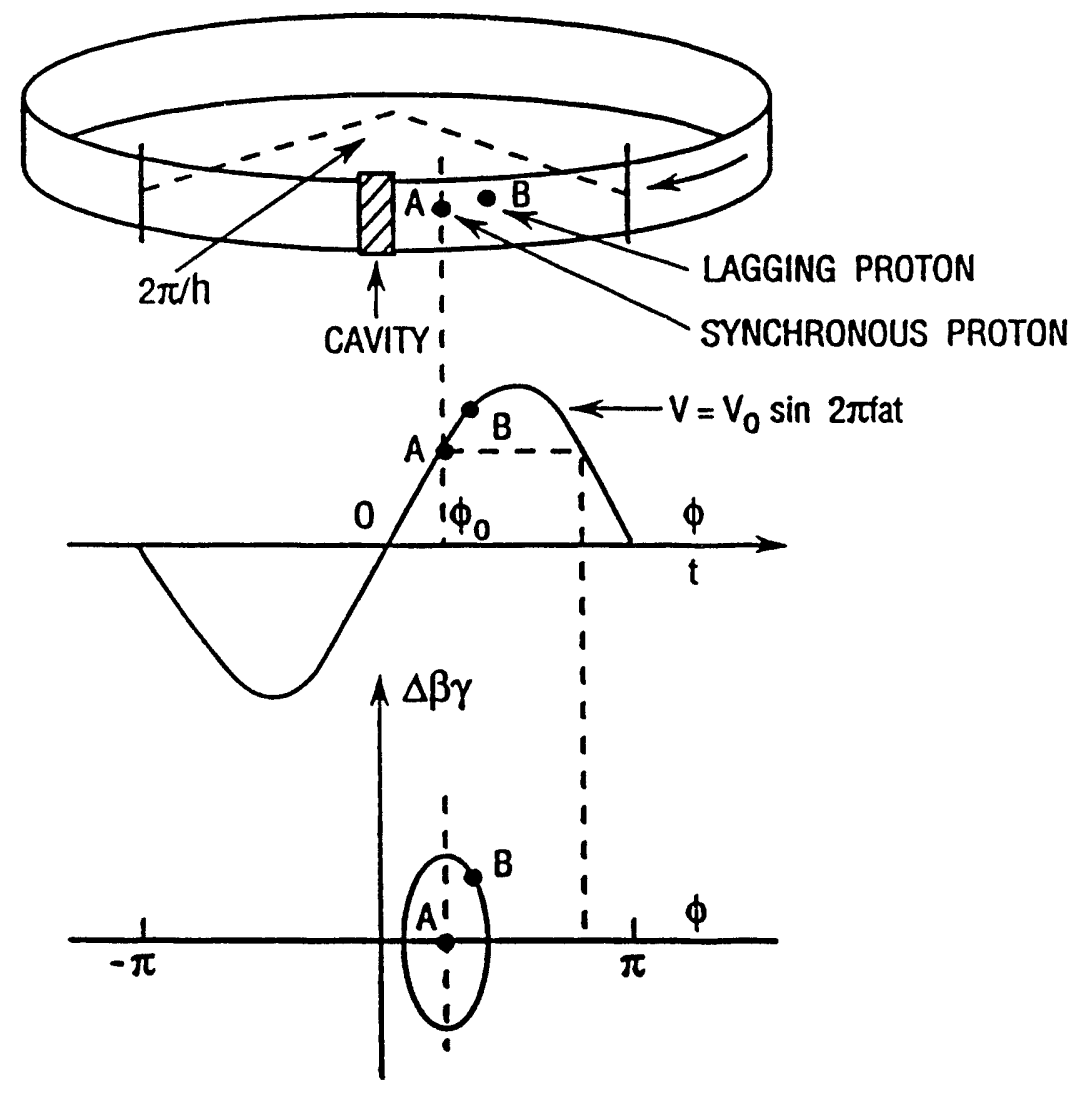

Fig. 5.1 Motion of non-synchronous particle relative to that of synchronous particle.

Particles in the vicinity of the synchronous particle are called non-synchronous particles (n.s.p.). Parameters characterizing the n.s.p. with small deviations from the s.p. are given by 


$$
\begin{aligned}
R=R_{0}+\Delta R, & \phi=\phi_{0}+\Delta \phi, \\
p=p_{0}+\Delta p, & E=E_{0}+\Delta E, \\
\theta=\theta_{0}+\Delta \theta, & \Omega=\Omega_{0}+\Delta \Omega .
\end{aligned}
$$

Here $\theta$ is the azimuth around the accelerator circumference $(0 \leq$ $\theta \leq 2 \pi), \phi$ is the phase of the rf field, and $\Omega$ is the revolution frequency around the synchrotron. Then

$$
\begin{aligned}
\Delta \phi & =-h \Delta \theta, \\
\Delta \Omega & =\frac{d}{d t} \Delta \theta=-\frac{1}{h} \frac{d \phi}{d t} .
\end{aligned}
$$

The fractional change of the revolution frequency relative to the fractional momentum change is called the frequency slip factor $\eta$

$$
\eta \equiv-\frac{\Delta \Omega / \Omega}{\Delta p / p}=\frac{1}{\gamma_{t r}^{2}}-\frac{1}{\gamma^{2}},
$$

where $\gamma_{t r}$ is the transition energy of the synchrotron, to be defined later. It is roughly equal to the horizontal betatron tune for a strong-focusing machine. If

$$
\gamma<\gamma_{t r}, \text { then } \Omega\left(p_{0}+\Delta p\right)>\Omega\left(p_{0}\right),
$$

while if

$$
\gamma>\gamma_{t r}, \text { then } \Omega\left(p_{0}+\Delta p\right)<\Omega\left(p_{0}\right) .
$$

Note that in the latter case, a higher energy particle circulates more slowly than the s.p. - the higher energy particles lag behind. The equation of motion for a n.s.p. is

$$
\begin{aligned}
\frac{d}{d t}\left(\frac{\Delta E}{\Omega_{0}}\right) & =\frac{e \bar{V}}{2 \pi}\left[\sin \phi-\sin \phi_{0}\right], \\
\frac{d \phi}{d t} & =\frac{h \eta \Omega_{0}}{p_{0} R_{0}}\left(\frac{\Delta E}{\Omega_{0}}\right),
\end{aligned}
$$

or the equivalent second-order differential equation

$$
\frac{d^{2}}{d t^{2}}(\Delta \phi)-\frac{e \bar{V} \eta \Omega_{0} \cos \phi_{0}}{2 \pi p_{0} R_{0}}(\Delta \phi)=0
$$




\subsection{Phase Stability}

From Eq. (5.12), stable oscillation requires

$$
\eta \cos \phi_{0}<0 \text {. }
$$

(a) Below transition $\eta<0$, hence $0<\phi_{0}<\pi / 2$.

(b) Above transition $\eta>0$, hence $\pi / 2<\phi_{0}<\pi$.

See Fig. 5.2.
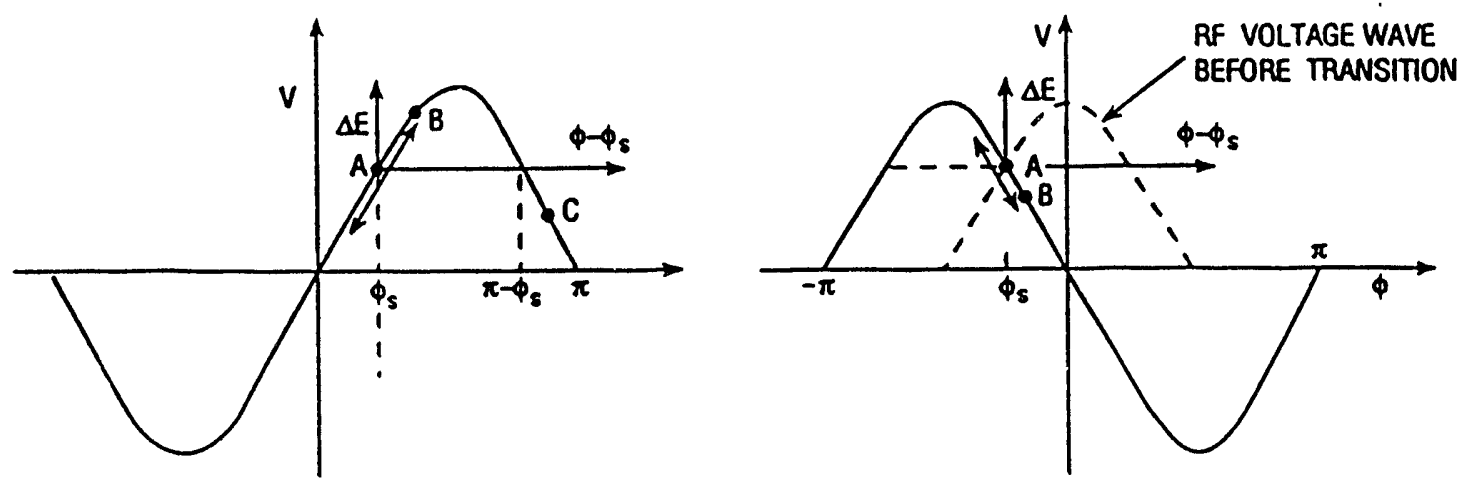

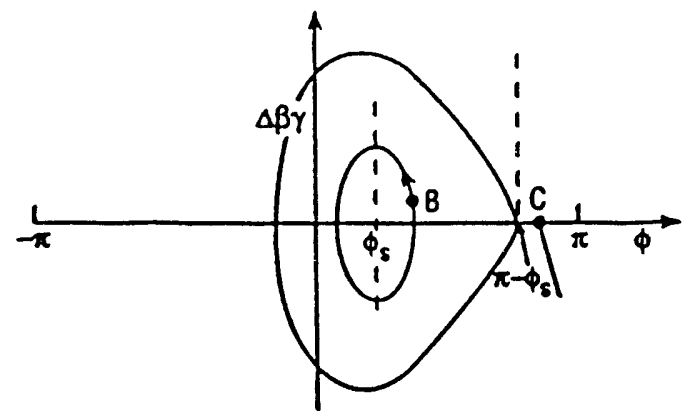

(a)

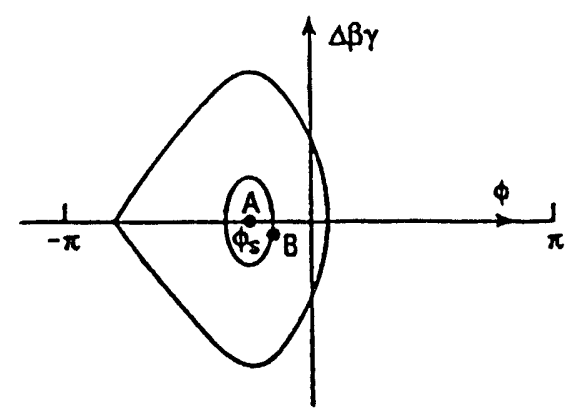

(b)

Fig. 5.2 Relationship of synchronous phase to if voltage wave below and above transition.

For a positive coefficient, the synchrotron oscillation is stable and the frequency of the small-amplitude oscillations $\Omega_{s}$ is given by

$$
\Omega_{s}=\left[-\frac{e \bar{V} \eta \Omega_{0} \cos \phi_{0}}{2 \pi p_{0} R_{0}}\right]^{1 / 2}
$$


and

$$
\frac{d^{2}(\Delta \phi)}{d t^{2}}+\Omega_{s}^{2}(\Delta \phi)=0
$$

Hence, the synchrotron tune of the oscillations is

$$
\nu_{s}=\frac{\Omega_{s}}{\Omega_{0}} .
$$

Typically, $\nu_{s} \ll 1$, whereas $\nu_{x, y} \gg 1$; the betatron tunes are much larger than unity.

\subsection{Transition Energy}

Assume that $\tau$ is the time to complete one revolution of the circumference $C$ at speed $v$. Then $\tau=C / v$ and

$$
\frac{\partial \tau}{\tau}=\frac{\delta C}{C}-\frac{\delta v}{v}
$$

From

$$
\frac{\delta C}{C}=\alpha \frac{\delta p}{p}, \quad \frac{\delta v}{v}=\frac{1}{\gamma^{2}} \frac{\delta p}{p}
$$

we have

$$
\frac{\delta \tau}{\tau}=\left(\alpha-\frac{1}{\gamma^{2}}\right) \frac{\delta p}{p} \equiv\left(\frac{1}{\gamma_{t r}^{2}}-\frac{1}{\gamma^{2}}\right) \frac{\delta p}{p}=\eta \frac{\delta p}{p},
$$

which implies that, for a particle with energy larger than the transition energy, the increase of path length due to the dispersion function is larger than the speed increase. As $\eta \rightarrow 0, \Omega_{s} \rightarrow 0$ also, which implies there is no phase stability. When accelerating protons through $\gamma_{t r}$, the RF phase must be switched abruptly from $\phi_{0}$ to $\pi-\phi_{0}$ to keep phase stability.

Exercise We saw, using the smooth approximation, that $\alpha \simeq \nu^{-2}$. Use this and the above relation between $\alpha$ and $\gamma_{t r}$ to show that $\gamma_{t r} \simeq \nu$. This justifies the statement above that $\gamma_{t r}$ is roughly equal to the horizontal betatron tune in a synchrotron. 
As the beam approaches transition, $\eta$ approaches zero, which means that the particles are locked into a particular phase relative to the rf wave. As a particular consequence, close to transition the particles constituting the bunch cannot follow as the rf bucket shape changes rapidly. This results in a so-called non-adiabatic time during which the concept of an RF bucket has no meaning. (See Holmes [6] for more details on the this topic.) This concept was recognized in the very early days by Courant and Snyder [3]. The non-adiabatic time is given by

$$
T_{\mathrm{na}}=\left(\frac{\pi \beta_{t r}^{3} \gamma_{t r}^{3}}{h \Omega_{0}^{2} \dot{\gamma}} \frac{E_{t r} / e}{V_{r f} \cos \phi_{0}}\right)^{1 / 3} .
$$

The path length for a particle to complete one revolution around the accelerator can be expressed as

$$
\frac{\Delta C}{C_{0}}=\alpha_{0} \frac{\Delta p}{p_{0}}+\alpha_{1}\left(\frac{\Delta p}{p_{0}}\right)^{2}+\cdots
$$

where $\alpha_{0}$ is the momentum compaction factor discussed earlier, and $\alpha_{1}$ gives the second derivative. The Johnsen effect arises from the chromatic nonlinearity represented by a nonzero $\alpha_{1}$ in Eq. (5.21). We will write down the expression for the transition gamma $\gamma_{t r}$ of different particles in the beam to second order in $\Delta p / p_{0}$. In doing so we need to make sure we are always defining $\gamma_{t r}$ in terms of local derivatives, rather than differences from the nominal circumference $C_{0}$ :

$$
\frac{1}{\gamma_{t r}^{2}}=\frac{p}{C} \frac{d C}{d p}
$$

After taking appropriate derivatives and some algebra, we obtain the variation of $\gamma_{t r}$ with $\Delta p / p_{0}$ :

$$
\gamma_{t r}=\gamma_{t r, 0}\left[1-\frac{1}{2}\left(1+\frac{2 \alpha_{1}}{\alpha_{0}}-\alpha_{0}\right) \frac{\Delta p}{p_{0}}\right] .
$$

Exercise Derive Eq. (5.23). Note that $\gamma_{t r, 0}^{-2}=\left(p_{0} / C_{0}\right) d C / d p=$ $\alpha_{0}$. 
The variation of $\gamma_{t r}$ over the particles making up the beam, coupled with their different energies, means that the particles within the beam do not all cross transition at the same time. We define the Johnsen or nonlinear time $T_{J}$ as the time difference between passage through transition of a particle at $\Delta p / p_{0}=0$ and $\Delta p / p_{0}=\sigma_{p} / p_{0}$. One can show that

$$
T_{J}=\frac{\gamma_{t r, 0}}{\dot{\gamma}}\left(\frac{3}{2}+\frac{\alpha_{1}}{\alpha_{0}}-\frac{\alpha_{0}}{2}\right) \frac{\sigma_{p}}{p_{0}} .
$$

Exercise Derive Eq. (5.24).

A problem in the transmission of beams through transition can arise if the nonlinear time is larger than the non-adiabatic time. In this case some particles in the beam will be above, while others will be below, transition for a period of time during which the motion of particles in phase space is describable by the standard set of trajectories, i.e. some particles will be found on bounded and others on unbounded trajectories. This situation will persist until the non-adiabatic time is entered and the particles become locked onto a certain rf phase. A semi-analytic description of the longitudinal emittance dilution arising from the Johnsen effect can be parameterized approximately as

$$
\frac{\Delta E}{E_{0}}= \begin{cases}0.76 T_{J} / T_{\mathrm{na}} & T_{J} \ll T_{\mathrm{na}}, \\ e^{0.43\left(T_{J} / T_{\mathrm{na}}\right)}-1 & T_{J}>T_{\mathrm{na}} .\end{cases}
$$

Methods to alleviate the transition effects have been reviewed by Holmes [6].

\subsection{Hamiltonian Formulation}

If we introduce the energy variable $W$, canonically conjugate to the position variable $\phi$, where

$$
W \equiv \Delta E / \Omega_{0}
$$


then the synchrotron oscillation equations (5.10) and (5.11) can be derived from the Hamiltonian

$$
H=\frac{h \eta \Omega_{0}}{2 p_{0} R_{0}} W^{2}+\frac{e \bar{V}}{2 \pi}\left[\cos \phi-\cos \phi_{0}+\left(\phi-\phi_{0}\right) \sin \phi_{0}\right] .
$$

Exercise Derive Eqs. (5.10) and (5.11) from Eq. (5.27).

For small amplitudes, we can simplify the above expression to

$$
H \simeq \frac{h \eta \Omega_{0}}{2 p_{0} R_{0}} W^{2}-\frac{e \bar{V}}{2 \pi} \cos \phi_{0} \frac{(\Delta \phi)^{2}}{2}
$$

which yields Eq. (5.12) and shows that the constant Hamiltonian carve in $(\Delta \phi, W)$ space is an ellipse for small amplitudes. In this phase space, $\phi=\phi_{0}$ is a stable fixed point, $\phi_{u}=\pi-\phi_{0}$ is an unstable fixed point. The boundary is the separatrix. See Fig. 5.3.

Exercise Derive Eq. (5.28) from Eq. (5.27).

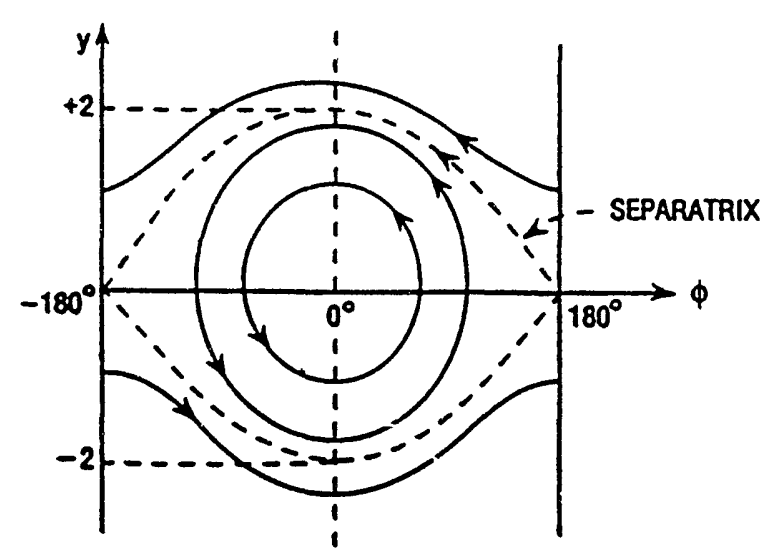

STATONURY BUCKET

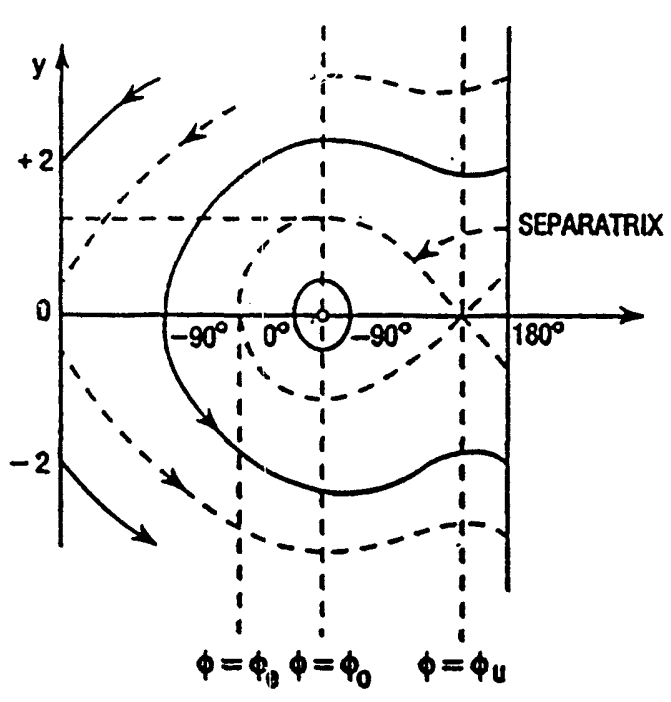

MOMNG BUCKET

Fig. 5.3 Longitudinal phase space with sketches of trajectories, fixed points, and separatrix. 
For a particle inside the stable region, the limits of oscillation in $W$ are given by $\dot{W}=0$, for which Eq. (5.10) gives two solutions, the stable fixed point $\phi=\phi_{0}$ and $\phi=\pi-\phi_{0} \equiv \phi_{u}$, the second solution corresponding to the unstable fixed point (UFP). The first solution, substituted into Eq. (5.27), gives the value of the Hamiltonian for the trajectory having an extremum $\bar{W}$ in amplitude, and we can write

$H=\frac{h \eta \Omega_{0} \widehat{W}^{2}}{2 p_{0} R_{0}}=\frac{h \eta \Omega_{0}}{2 p_{0} R_{0}} W^{2}+\frac{2 \bar{V}}{2 \pi}\left[\cos \phi-\cos \phi_{0}+\left(\phi-\phi_{0}\right) \sin \phi_{0}\right]$.

The extrema in the phase $\phi$ are given by $W=0$. Let us look at two interesting special cases.

Case 1: For stationary buckets $\left(\eta<0, \phi_{0}=0\right)$, putting $W=0$ into Eq. (5.2 $\mathrm{g})$ yields

$$
\frac{h \eta \Omega_{0}}{2 p_{0} R_{0}}{\bar{W}^{2}}^{2}=\frac{e \bar{V}}{2 \pi}[\cos \phi-1]=-\frac{e \bar{V}}{2 \pi} \sin ^{2} \frac{\phi}{2},
$$

which in turn gives the limits of the amplitudes in phase

$$
\phi_{\ell}= \pm 2 \sin ^{-1}\left[\frac{\pi h(-\eta) \Omega_{0}}{2 e \bar{V} p_{0} R_{0}} \widehat{W}^{2}\right]^{1 / 2} .
$$

Case 2: For a non-stationary bucket in the small-amplitude approximation, putting $\Delta \phi=\phi-\phi_{0} \ll 1$, Eq. (5.29) at $W=0$ yields

$$
\Delta \widehat{\phi}= \pm\left[\frac{2 \pi h(-\eta) \Omega_{0}}{2 e \bar{V} p_{0} R_{0} \cos \phi_{0}}\right]^{1 / 2} \widehat{W}
$$

The ratio $\widehat{W} / \Delta \widehat{\phi}$ is an important parameter in matching bunches of particles into rf buckets, for example when transferring a beam from one accelerator to another.

We have already seen that the separatrix limiting the phasestable region terminates at the UFP $W=0, \phi_{u}=\pi-\phi_{0}$. We can therefore substitute these value into Eq. (5.29) to determine the extreme amplitudes in $W$ of the separatrix. In other words, these extrema $W_{\text {sep }}$ are given by

$$
\frac{h \eta \Omega_{0}}{2 p_{0} R_{0}} W_{\text {sep }}^{2}=\frac{e \bar{V}}{2 \pi}\left[-2 \cos \phi_{0}+\left(\pi-2 \phi_{0}\right) \sin \phi_{0}\right] .
$$


Exercise Derive Eq. (5.33).

The other extremum of phase, $\phi_{e}$, is obtained by using Eq. (5.33) in Eq. (5.29) with $W=0$. Then

$$
\cos \phi_{e}-\phi_{e} \sin \phi_{0}=\left(\pi-\phi_{0}\right) \sin \phi_{0}-\cos \phi_{0} \text {. }
$$

Exercise Derive Eq. (5.34).

This transcendental equation in $\phi_{e}$ reduces to $\cos \phi_{e}=-1,+1$ for $\phi_{0}=0, \pi$, and thus yields the adjacent UFP, as expected for stationary buckets.

Another useful quantity to be derived from Eq. (5.29) is the concept of longitudinal emittance, or phase-space area of a bunch of particles. In the small-amplitude approximation, Eq. yields

$$
H=\frac{h \eta \Omega_{0}}{2 p_{0} R_{0}} W^{2}-\frac{e \bar{V}}{2 \pi} \cos \phi_{0} \frac{(\Delta \phi)^{2}}{2}=-\frac{e \bar{V}}{2 \pi} \cos \phi_{0} \frac{(\Delta \hat{\phi})^{2}}{2} .
$$

In terms of the bunch length $\Delta \hat{\phi}$, Eq. (5.35) becomes

$$
\frac{(\Delta \phi)^{2}}{(\Delta \widehat{\phi})^{2}}-\frac{h \eta \Omega_{0}}{2 p_{0} R_{0}} \frac{4 \pi}{e \bar{V} \cos \phi_{0}(\Delta \widehat{\phi})^{2}} W^{2}=1,
$$

which is the standard equation of an ellipse. The area of the phasespace ellipse is given by

$$
A_{s}=\pi(\Delta \hat{\phi})\left[\frac{e \bar{V} \cos \phi_{0} p_{0} R_{0}}{2 \pi h(-\eta) \Omega_{0}}\right]^{1 / 2} .
$$

This parameter, or $A_{s} / \pi$, the longitudinal emittance of the beam, stays constant during the acceleration cycle and is an important parameter governing the beam quality in a synchrotron or storage ring. In terms of $\widehat{W}$ and $\Delta \widehat{\phi}$, the area is $A_{s}=\pi \widehat{W} \Delta \hat{\phi}$. Since $A_{s}$ is an invariant quantity during acceleration, it is often convenient to express $\widehat{W}$ or $\Delta \widehat{\phi}$ in terms of $A_{s}$. 


\subsection{Adiabatic Damping}

Over the acceleration cycle, the energy spread $\widehat{W}$ and phase extent $\Delta \widehat{\phi}$ of the beam obey the following relation:

$$
\begin{aligned}
\widehat{W} & \propto\left(-\frac{p_{0} R_{0} \bar{V} \cos \phi_{0}}{\eta \Omega_{0}}\right)^{1 / 4}, \\
\Delta \widehat{\phi} & \propto\left(-\frac{\eta \Omega_{0}}{p_{0} R_{0} \bar{V} \cos \phi_{0}}\right)^{1 / 4}
\end{aligned}
$$

therefore $\Delta \hat{\phi}$ decreases with the $1 / 4$ power of $p_{0}$. The longitudinal emittance $A_{s} / \pi=\widehat{W} \Delta \widehat{\phi}$ has dimensions of energy $\times$ time, and is generally measured in units of $\mathrm{eV}$-sec. Note that this time there is no need to introduce a normalized emittance, because $p_{0}$ has not been scaled out of the emittance: the above definition is adiabatically invariant under acceleration. Figure 5.4 shows the energy dependence of $\Delta E, \Delta \phi$, and $\Delta R$ in a typical synchrotron.

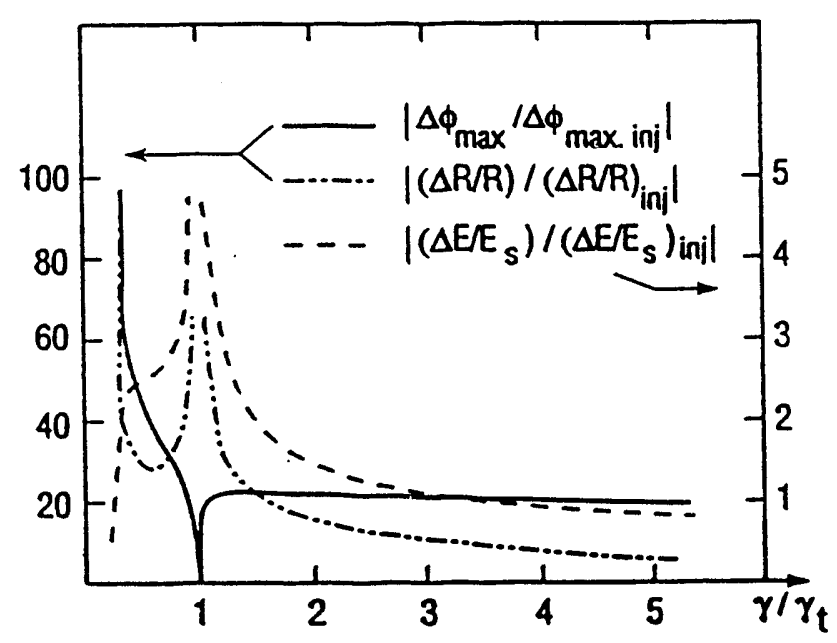

Fig 5.4 Typical variation of $\Delta \phi, \Delta R$, and $\Delta E$ as the beam energy increases.

\subsection{Phase-space Matching during Synchronous Transfer}

We have already seen that matching between two optical channels is important for transverse emittance preservation. The same is certainly true in longitudinal phase space. The matching can be achieved if 


$$
\begin{aligned}
\left(\frac{\widehat{W}}{\Delta \widehat{\phi}}\right)_{B} & =\left(\frac{\widehat{W}}{\Delta \widehat{\phi}}\right)_{A} \\
\Rightarrow \quad\left(\frac{h \Omega_{0} \cos \phi_{0}}{\eta}\right)_{B} & =\left(\frac{h \Omega_{0} \cos \phi_{0}}{\eta}\right)_{A} .
\end{aligned}
$$

If two machines do not satisfy the matching condition, some intermediate step before transfer must be implemented to achieve the matched condition. The matched condition is often achieved by adiabatically varying the voltage on the first, lower energy accelerator. The beam loading instability, however, may make it difficult to control the phase and amplitude of the rf voltage when it is low. With bunch rotation, matched beams can be created in the first machine at a much higher voltage and in a shorter period, as illustrated in Fig. 5.5.

(a)

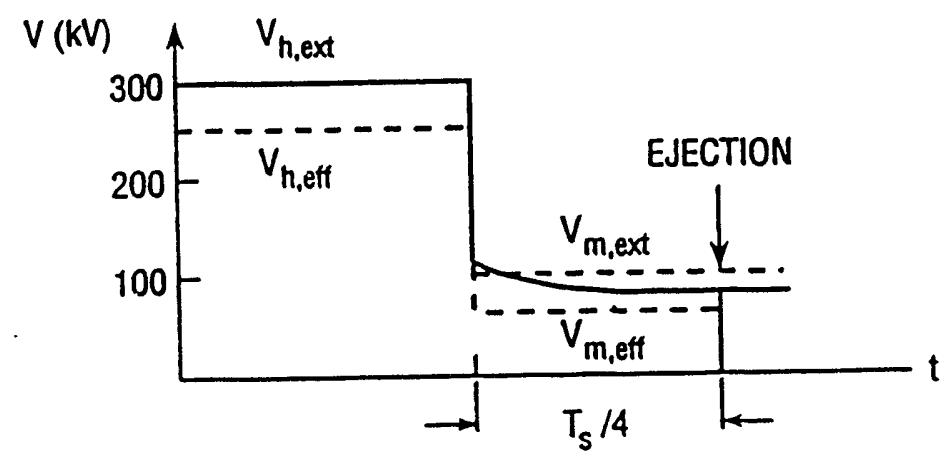

(b)

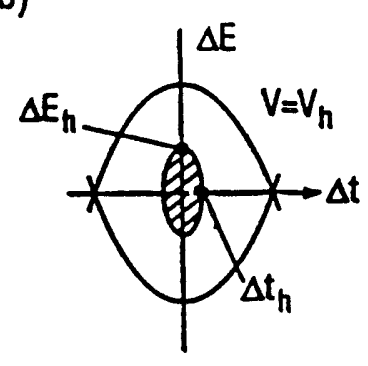

MATCHED BUNCH

BUNCH ROTATION MATCHING

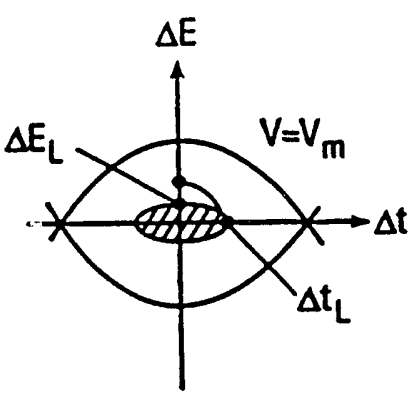

AFTER 1/4 TURN IN MISMATCHED BUCKET

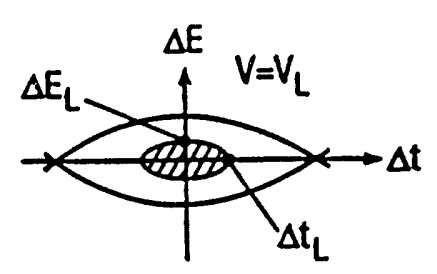

EQUIVALENT MATCHED BUCKET

Fig. 5.5 Matching of a beam to a synchrotron in longitudinal phase space, to avoid longitudinal emittance blowup. 


\section{Bibliography}

1. For the historical development of high energy accelerators, M.S. Livingston, "The Development of High Energy Accelerators," Dover (1966).

2. For new major accelerator projects in progress, L.C. Teng, "Accelerator Projects, World," in Physics of Particle Accelerators, AIP Conf. Proc. 184, p. 2116 (1989).

3. For introduction to the accelerator physics of synchrotrons, D.A. Edwards and M.J. Syphers, "An Introduction to the Physics of Particle Accelerators," Ibid. p. 2.

M. Sands, "The Physics of Electron Storage Rings - An Introduction," SLAC-121 (1970).

E. Courant and H. Snyder, "Theory of the Alternating Gradient Synchrotron," Ann. Phys. 3, 1 (1958).

4. For phase-space dynamics,

A. Lichtenberg, "Phase-Space Dynamics of Particles," Wiley (1969).

C. Bovet, "Phase-Space Dynamics for Accelerators," CERN Academic Training Program \#52 (1971).

E. Courant, R. Ruth and W.T. Weng, "Stability in Dynamical Systems I," in Physics of High Energy Accelerators, AIP Conf. Proc. 127, p. 294 (1985).

G. Guignard, "Phase-Space Dynamics," in Frontiers of Particle Beams, Springer-Verlag, pp. 3-50 (1988).

5. For detailed discussions and examples of beam emittance,

D. Finley, "Emittances through the Fermilab Accelerator Chain," in Physics of Particle Accelerators, AIP Conf. Proc. 184, p. 1910 (1989).

C. Lejeune and J. Aubert, "Emittance and Brightness: Definition and Measurements," in Adv. Elec. and Elec. Phys., Supp. 13a, 159 (1980).

6. For longitudinal dynamics,

W.T. Weng, "Fundamentals - Longitudinal Motion," in AIP Conf. Proc. 184, pp. 243-287 (1989); BNL 42195 (1988).

G. Dôme, "Theory of RF Acceleration," in 1985 CERN Accelerator School, CERN 87-03, pp. 110-158.

S.D. Holmes, "Low Energy Aspects of Circular Accelerators," FNAL-Conf-90/275 (1990). 

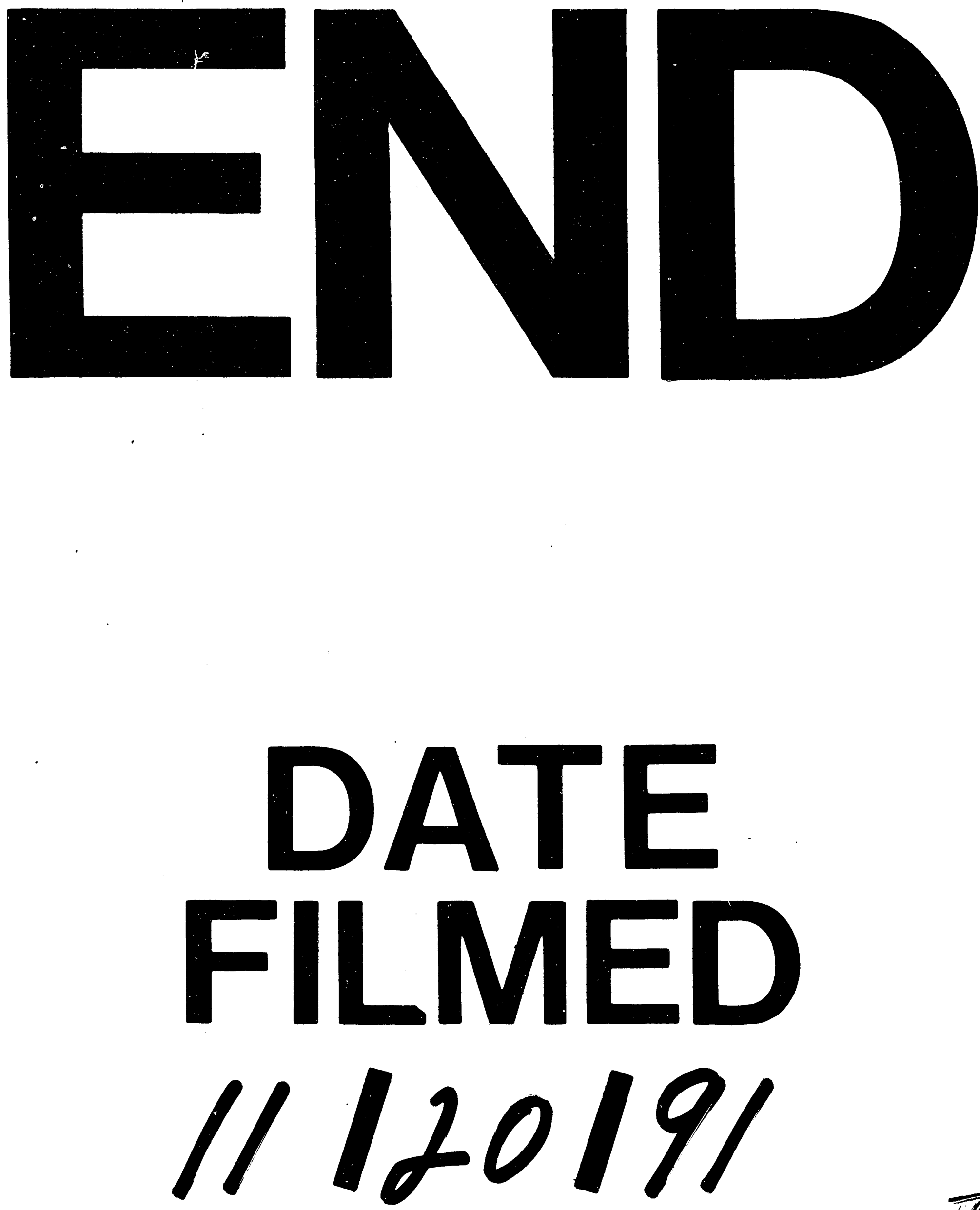

II 
